\title{
Microcarriers' suspension and flow dynamics in orbitally shaken bioreactors
}

\author{
Pieralisi I. ${ }^{a}$, Rodriguez G. ${ }^{b}$, Micheletti M. ${ }^{b}$, Paglianti A. ${ }^{\text {a }}$, Ducci A. ${ }^{\mathrm{c}}$ \\ ${ }^{a}$ Dipartimento di Ingegneria Civile, Chimica, Ambientale e dei Materiali, Universitá di Bologna, Via Terracini \\ 28, 40131, Bologna, Italy \\ ${ }^{b}$ University College London, Department of Biochemical Engineering, WC1E 7JE, UK \\ ${ }^{c}$ University College London, Department of Mechanical Engineering, WC1E 7JE, UK
}

\section{Abstract}

In the present work an effort is made to determine the suspension speed of microcarriers in an orbitally shaken bioreactor of cylindrical geometry, and to assess the associated two-phase flow by means of Particle Image Velocimetry (PIV). Microcarrier technologies are commonly used in the bioprocess industry to culture adherent-dependent cells in three dimensional flow. Commercial GE Cytodex microcarriers were employed throughout this study to best mimic the flow conditions occurring in a bioreactor under standard operating conditions. Suspension speed measurements were obtained at different solid concentrations, that are typical for cell cultures, and for different combinations of orbital to cylinder diameters' ratio, $d_{o} / d_{i}(c=2.5-12.5 \mathrm{~g} / \mathrm{L}$; $\left.d_{o} / d_{i}=0.2-0.7 ; N=0-200 \mathrm{RPM}\right)$. The current two-phase PIV results show that mean flow dynamics occurring in the cylindrical bioreactor are not significantly affected by the presence of the microcarriers, and that their suspension is directly associated to the flow transition reported by Weheliye et al. (2013). The flow scaling law included in their study can be successfully employed to predict the full suspension speed across bioreactors of different scales and working under different operating conditions (i.e. inner diameter of the cylinder, $d_{i}$, orbital diameter, $d_{o}$, and filling volume, $\left.V_{f}\right)$.

Keywords: Orbitally shaken bioreactor, microcarriers' suspension speed, PIV, two-phase flow.

\section{Introduction}

Stem cells represent attractive therapeutic agents for a wide range of diseases due to their capacity to differentiate into a specialized cell type. The large number of cells required for clinical trials (up to millions cells/ $\mathrm{kg}$ of body weight) demands a fast and reproducible expansion protocol. Stem cells are adherent-dependent cells, as they are able to grow and differentiate only if attached to an appropriate support. Two-dimensional (2D) static culture methods rely on the use of disposable multi-layer vessels and have rapidly become the most common route for stem cells expansion (Simaria et al., 2014). However, these methods do not seem appropriate for stem cell large scale production because of the limited cell productivity, labor intense handling procedures and long cultivation times. For example, recent studies proved that commercial requirements would be satisfied only with the production of up to $10^{13}$ cells per batch, and the use of $10^{5}$ layered vessels per lot, which is not a feasible process (Simaria et al., 2014). In 
addition, these systems are not able to supply reproducible batch culture conditions (Mohamet et al., 2010). A cost-effective approach which has demonstrated to overcome many of the limitations of 2D cultures is represented by three-dimensional (3D) dynamic culture methods based on microcarriers suspension technologies (Frauenschuh et al., 2007; Sart et al., 2009; Storm et al., 2010). Microcarriers are generally spherical beads with an ideal size of 100-300 $\mu \mathrm{m}$, and can be made of different materials (plastics, glass, silica dextran, collagen). Cell attachment is promoted through electrical charges or collagen coating. In microcarriers culture cells grow as monolayers on the surface of the beads or as multilayers in the pores of macroporous structures, that are usually suspended in culture medium by gentle stirring (GE Healthcare Life Sciences, 2013). With this technique the physiological microenvironment of stem cells can be easily monitored and reproduced, with significant advantages towards large scale production (King and Miller, 2007; Liu et al., 2014). The use of microcarriers in cell cultures allows an increase in the surface area $(\mathrm{SA})$ per unit volume $\left(\mathrm{cm}^{2} / \mathrm{mL}\right)$, improving product consistency and decreasing costs (Frauenschuh et al., 2007; Sart et al., 2009; Schop et al., 2008, 2009; Ferrari et al., 2012). Most studies have focused on investigating the optimal medium components, the microcarrier type and concentration, however only a few considered the engineering aspects, the quality of the microcarriers suspension and their impact on the liquid phase flow and turbulence levels. Conditions that promote efficient attachment and uniform distribution of the cells over the microcarriers population must be sought and optimized, and from this point of view, the flow and mixing dynamics occurring in the bioreactor must be thoroughly investigated and carefully selected. Efficient flow dynamics is crucial to achieve complete suspension of the microcarriers, thus preventing particle agglomeration and enhancing the available adherence area for the cells, while mixing is essential to promote mass transfer within the environment and to avoid spatial gradients in culture parameters (e.g. dissolved gases, nutrient concentration, pH), that can directly affect cell growth (Lara et al., 2006). At laboratory scale, adherent-dependent cell cultures are often grown on microcarriers in orbitally shaken reactors (OSRs), which offer an effective solution in the early stages of bioprocess development. Once the process is optimized, it is then scaled-up to traditional stirred tank reactors (STRs), where the velocity characteristics and turbulence levels are different from those found in shaken cultures. To overcome the scaling up/down limitations due to the different types of bioreactor, current bioprocess strategies have seen the development of miniature stirred tanks (for example the Ambr15 cell culture, 10-15 $\mathrm{mL}$ ), to be employed in bioprocess development, while large scale shaken systems up to a scale of $1000 \mathrm{~L}$ have recently become available in the market, and studies have demonstrated their mixing effectiveness and oxygen transfer capabilities (Zhang et al., 2009).

Recently a few studies have focused on the mixing and fluid dynamics of shaken bioreactors. The works of Weheliye et al. (2013) and Ducci and Weheliye (2014) have provided a detailed understanding of the single-phase flow generated in an orbitally shaken bioreactor at different operating conditions (e.g. shaker rotational speed, $N$, and medium height inside the tank, $h$ ), geometrical characteristics (e.g. cylinder inner diameter, $d_{i}$, and orbital shaking diameter, $d_{o}$ ) and fluid viscosity, $\nu$. A Fr-Re flow transition map was derived, where four types of mean 
flow were identified depending on the combination of Froude and Reynolds numbers selected.

A transition from a toroidal to a precessional vortex configuration was detected with increasing Froude number, $\mathrm{Fr}$, for fluids of water-like viscosity close to those employed in cell culture (high Re range). At low $F r$ the free surface exhibited an elliptic shape in phase with the shaker table orbital movement, while an increasing degree of out-of-phase and a highly three-dimensional free surface characterised the high end of shaker speeds investigated (Weheliye et al., 2013). A flow scaling law was derived to predict the occurrence of this flow transition based on the Froude number, $F r$, the fluid non-dimensional height, $h / d_{i}$, and the orbital to cylinder diameter ratio, $d_{o} / d_{i}$. More specifically it was found that for $h / d_{i} \leq \sqrt{d_{o} / d_{i}}$ the critical Froude number can be obtained from Equation 1, and it is associated to the toroidal vortex reaching the bottom of the cylindrical bioreactor before transition occurs, while for $h / d_{i} \geq \sqrt{d_{o} / d_{i}}$ transition takes place without the toroidal vortex expanding all the way to the reactor bottom, and the critical speed/Froude number can be found from Equation 2.

$$
\begin{aligned}
F r_{d_{o}} & =\frac{1}{a_{o w}} \frac{h}{d_{i}}\left(\frac{d_{o}}{d_{i}}\right)^{0.5} \\
F r_{d_{i}} & =\frac{1}{a_{o w}}
\end{aligned}
$$

Where $a_{\text {ow }}$ is a constant depending on the fluid employed (1.4 for water), and the Froude number is defined as the ratio of the centrifugal to the gravitational accelerations, $F r_{d}=2 \pi^{2} N^{2} d / g$, with $d$ being either the orbital $\left(d=d_{o}\right.$, Equation 1$)$ or cylinder $\left(d=d_{i}\right.$, Equation 2$)$ diameters. The flow scaling law of Weheliye et al. (2013) was successfully applied to the mixing time experiments of Rodriguez et al. (2013, 2014) obtained by means of a base-acid colorisation technique in shaken bioreactors of cylindrical geometry. Rodriguez et al. (2014) compared their data to those obtained by Tissot et al. (2010) for very different operating conditions $\left(d_{o}, V_{f}\right)$ and bioreactor sizes $\left(d_{i}\right)$, and found out that the two sets of data scaled well when the mixing number was plotted against the ratio of $F r / F r_{c r}$, and achieved a constant value after flow transition occurred $\left(F r>F r_{c r}\right)$.

Recently Mancilla et al. (2015) compared the mean flow and turbulence levels in orbitally shaken flasks with conventional, coiled, 1 and 3 baffle geometries. The 2D-PIV results obtained on a horizontal plane of measurements for increasing rotational speed, $N$, indicate that the configuration with a single baffle is characterised by turbulence levels $25 \%$ higher than in the other configurations investigated, and should be employed for production of bacterial cultures. $\mathrm{Nu}-$ merical simulation studies of the flow dynamics in shaken systems have been carried out by Zhang et al. (2005) and Zhang et al. (2008) for 250-ml Erlenmeyer flasks and for 24-well and 96-well bioreactors with water-like viscous fluids, respectively, while Kim and Kizito (2009) simulated the flow in a cylindrical shaken bioreactor for different fluid viscosity. Discacciati et al. (2012) developed a pressure correction method to best capture the free surface deformation and assess the shear stress levels in an orbitally shaken cylindrical container for a high viscous fluid, while Reclari et al. (2014) compared the free surface wave measurements in a shaken cylinder against those predicted by a potential sloshing model, and identified the presence of different 
modal responses inducing different flow regimes.

Little information can be found in the literature regarding the flow and mixing dynamics taking place in bioreactors when microcarriers suspensions are considered. Collignon et al. (2010) investigated the suspension of microcarriers for TTP Mixel, A325-A320 Lightnin, three streamed-blades VMI-Rayneri, and Elephant Ear Applikon impellers in a stirred tank reactor, and compared the flow characteristics, shear rate and power consumptions of the different impellers at the corresponding just suspended speed, $N_{j s}$. Their results indicated that the TTP Mixel and the Ear Elephant Applikon impellers produced the lowest mechanical constraints at their just suspended speed. PIV measurements in a spinner flask were carried out by Ismadi et al. (2014) to assess to what extent flow shear stresses can affect cell culture of mouse induced pluripotent stem cells (iPSC) attached to microcarriers. They show that optimum number of cells was achieved over 7 days in 25 RPM suspension culture, corresponding to a maximum shear of $0.0984 \mathrm{~Pa}$. Nienow et al. (2014) developed a new method for the harvesting of human mesenchymal stem cell (hMSC) in a spinner flask. The cells were cultured in dimple-bottomed spinner flasks equipped with a magnetic horizontal stir bar and a vertical paddle at a working volume of $100 \mathrm{~mL}$ and at $30 \mathrm{RPM}\left(N_{J S}\right)$. After expansion, harvesting was implemented by adding trypsin-EDTA and agitating the microcarriers suspension for 7 mins at 150 RPM. Their study indicates that intense agitation for a short period ( 7 mins) under the presence of a suitable enzyme can promote cell detachment without damaging the cells or affecting their attributes. The overall harvesting efficiency was above $95 \%$.

Recently Olmos et al. (2015) determined the critical agitation speed for microcarriers' suspension in orbitally shaken Erlenmeyer flasks and cylindrical reactors. They stained the microcarriers with Trypan blue and used a camera rigidly moving with the shaker table to assess their suspension at increasing speed. The Vachy-Buckingham theorem was employed to obtain the non-dimensional model of Equation 3.

$$
\frac{N_{s}}{\sqrt{g / d_{o}}}=\sqrt{\frac{F r_{s}}{2 \pi^{2}}}=A\left(\frac{h}{d_{i}}\right)^{0.5}\left(\frac{d_{o}}{d_{i}}\right)^{0.25}\left(\rho^{*}\right)\left(\frac{d_{p}}{d_{i}}\right)^{-0.07}
$$

Where $A$ is a constant depending on the type of geometry used (1.39 for cylinder, 0.12 for Erlenmeyer flask), and $\rho^{\star}$ and $d_{p}$ are the relative density and diameter of the microcarriers, respectively. It should be noted that in Equation 3 they considered a Froude number which is defined as a velocity ratio, and it is related to the one defined in this work by the square root of $F r$. Direct comparison of Equations 1 and 3 shows that the critical Froude number, $F r_{c r}$, associated to the flow transition reported by Weheliye et al. (2013), is related to the suspension Froude number, $F r_{s}$, obtained from the model of Olmos et al. (2015), with the non-dimensional fluid height, $h / d_{i}$ and orbital to cylinder diameter ratio, $d_{o} / d_{i}$, terms having the same exponents. It is interesting to point out that their model showed a very good agreement also for Erlenmeyer flasks, implying that a similar flow transition to the one reported by Weheliye et al. (2013) could take place also in this geometry. 
In the present study a different approach has been developed, where the "just-suspended" speed is estimated from the light scattered by the microcarriers on a laser plane parallel to the bottom of the cylindrical bioreactor, while vertical plane measurements were obtained to assess the homogeneity of microcarriers across the tank volume. Furthermore, two-phase Particle Image Velocimetry experiments were carried out to better comprehend the flow and mixing dynamics in the presence of microcarriers, and to assess how their concentration affects the mean flow characteristics.

\section{Materials and methods}

Depending on the measurements being carried out, two different experimental rigs were employed. Figure 1 (a) shows the experimental set-up used to obtain the "just suspended speed", where a $300 \mathrm{~mW}$ continuous diode laser, a mirror, a Net iCube camera with Macro Lens, and a cylindrical bioreactor with a flat bottom, were all rigidly mounted on a Lab LS-X Kühner shaker table. The laser-light was directed horizontally in order to illuminate the plane located immediately over the vessel bottom, while a camera gained optical access to the measurement plane through a mirror located underneath the bioreactor. The camera was equipped with a macro lens with a shallow depth-of-field, that allowed to capture any small variation of the image brightness, which was directly related to the light scattered by the microcarriers sitting at the bottom of the bioreactor, as the shaking speed was varied. For each orbital speed investigated, 50 images were captured, and analysed by home-built Matlab routines to obtain a quantitative average result of the suspension conditions of the system. Before capturing a set of images a sufficient time was given to ensure steady-state condition was achieved at each speed investigated. Experiments were carried out in a borosilicate glass cylindrical bioreactor of size $d_{i}=7$ $\mathrm{cm}$, for different ranges of orbital diameters, $d_{o}=1.5-5 \mathrm{~cm}$, and shaker speeds, $N=60-140$ RPM. The working liquid was distilled water with a fluid height $h=3$ and $5 \mathrm{~cm}\left(V_{f}=115.5\right.$, $192.5 \mathrm{~mL})$. Commercial microcarriers, GE Cytodex $1\left(\rho=1.03 \mathrm{~kg} / \mathrm{L}, d_{50}=190 \mu \mathrm{m}\right)$ and GE Cytodex $3\left(\rho=1.04 \mathrm{~kg} / \mathrm{L}, d_{50}=175 \mu \mathrm{m}\right)$, were employed at concentrations typically adopted for stem cell cultures: $2.5,7.5,12.5 \mathrm{~g} / \mathrm{L}(0.25,0.75,1.25 w t \%)$. Their settling velocity was approximately $0.6 \mathrm{~mm} / \mathrm{s}$. More information on the characteristics of the microcarriers employed can be obtained in GE Healthcare Life Sciences (2013).

The two-phase PIV system is shown in Figure 1 (b), where a larger Kühner shaker table $(1 \times 1$ $\mathrm{m}^{2}$, SR200-X shaker) is used to hold two cameras sharing the same field of view by a $50 \%$ transmission/50 \%-reflection mirror and an optical guiding arm shining the laser onto a mirror positioned underneath the reactor. Contrary to the suspension speed experiments, in this case the measurement region consisted on the vertical plane bisecting the bioreactor into two halves. Each camera was equipped with a different light filter (either green, $\lambda=532 \mathrm{~nm}$, or orange $\lambda$ $=570 \mathrm{~nm}$ ) to distinguish between the solid and liquid phases. To improve the image quality of the solid phase, fluorescent Rhodamine B isothiocyanate was employed to stain GE Cytodex 3 
microcarriers, by exploiting the strong bond occurring between the dye and the thin collagen layer that coats the microcarriers' surface. The staining protocol consisted in mixing $2 \mathrm{mg}$ of Rhodamine in $50 \mathrm{ml}$ of deionized water for a $200 \mathrm{mg}$ sample of GE Cytodex 3. Staining was done at room temperature for $12 \mathrm{hrs}$ and a $45 \mu \mathrm{m}$ sieve was used to filter the stained particles. After this procedure the two-phase measurements could be carried out up to a solid concentration of $0.75 \mathrm{~g} / \mathrm{L}(0.075 w t \%)$. Above this threshold the image quality decreased due to the laser attenuation across the measurement plane induced by the presence of the microcarriers. Distilled water seeded with 1-40 $\mu \mathrm{m}$ flakes of painting was used as the continuous phase. Experiments were performed in a glass cylindrical bioreactor of size $d_{i}=10 \mathrm{~cm}$, with an orbital diameter, $d_{o}=5 \mathrm{~cm}$, and a fluid height $h=5 \mathrm{~cm}\left(V_{f}=392 \mathrm{~mL}\right)$ for different shaker speeds, $N=80-130$ RPM.

Phase-locked measurements were obtained by a magnetic encoder coupled to the Kühner shaker table. The origin of the angular coordinate, $\phi$, was set when the system reaches its position furthest to the left as the clockwise orbit is viewed from above. To fully resolve the large scale flow structures the measurement spatial resolutions of the liquid and solid phases were $\Delta x_{i}=1.66$ $\mathrm{mm}$ and $1.84 \mathrm{~mm}$, respectively, while the time interval between PIV image pairs was $\Delta t=1-2 \mathrm{~ms}$. The time interval, $\Delta t$, was selected according to the optimisation protocol developed by Gomez et al. (2010). In the rest of the article a cylindrical coordinate system $r, \phi, z$ is employed with the origin positioned on the cylinder axis at the bioreactor base. As mentioned in the introduction the Froude number based on the orbital diameter is an essential parameter to control the flow dynamics inside the bioreactor, and will be referred to here after either as $F r_{d_{o}}$ or, to simplify, as Fr. A comprehensive list of the operating conditions investigated for the suspension speed and PIV experiments is provided in Table 1.

\begin{tabular}{|l||l|}
\hline SUSPENDED SPEED & SOLID-LIQUID PIV \\
\hline$d_{i}=7 \mathrm{~cm}$ & $d_{i}=10 \mathrm{~cm}$ \\
\hline$d_{o}=1.5,2,2.5,3,4,5 \mathrm{~cm}$ & $d_{o}=5 \mathrm{~cm}$ \\
\hline$N=0-200 \mathrm{RPM}$ & $N=80,90,96,110,130 \mathrm{RPM}$ \\
\hline$h=2,3,4,5 \mathrm{~cm}\left(V_{f}=76.9-192.5 \mathrm{~mL}\right)$ & $h=5 \mathrm{~cm}\left(V_{f}=392.5 \mathrm{~mL}\right)$ \\
\hline$c=2.5,7.5,12.5 \mathrm{~g} / \mathrm{L}(0.25,0.75,1.25 w t \%)$ & $c=0.25,0.5,0.75 \mathrm{~g} / \mathrm{L}(0.025,0.05,0.075 w t \%)$ \\
\hline
\end{tabular}

Table 1: Geometrical details of the shaken systems and operational conditions investigated for the two-phase measurements.

\section{Results and discussion}

In the following sub-sections the three parts of the investigation, that is, microcarriers' suspension speed ( $\S 3.1$ ), microcarriers' dispersion ( $\S 3.2$ ), and two-phase flow dynamics $(\S 3.3)$, are 
discussed in sequence. In brief, the rationale for the selection of these three parts of the work was to identify the range of speeds over which suspension occurs for different operating conditions, to assess the microcarriers' suspension and dispersion mechanisms as the shaker speed is increased, and to determine the flow dynamics and transition of the two-phase system as well as compare them against those obtained for a single-phase (Weheliye et al., 2013).

\subsection{Microcarriers suspension speed}

The just suspended speed was estimated from the brightness of the images taken on the horizontal measurement plane, which is directly proportional to the amount of particles sitting at the bottom of the reactor. The image brightness, $I_{B}(N)$, at a given shaking speed, $N$, is defined in Equation 4 by adding the pixel greyscale, $p_{i j}$, across the area delimited by the bioreactor walls on the horizontal plane of measurement:

$$
I_{B}=\sum_{N_{t o t}} p_{i j}
$$

where $N_{\text {tot }}$ is the total number of pixels across the area.

The microcarriers' suspension process and its correlation to the brightness percentage index, $I_{B}(N) / I_{B}(0)$, for increasing shaking speed, $N$, can be gained from Figure 2, where steady-state images of the microcarriers' concentration over horizontal planes are coupled to the $I_{B}(N) / I_{B}(0)$ curve at key speeds. This set of experiments was carried out for an orbital diameter $d_{o}=2.5$ $\mathrm{cm}$ and a microcarriers' concentration $c=2.5 \mathrm{~g} / \mathrm{L}$. At low shaking speeds the microcarriers are uniformly distributed over the vessel bottom, and the brightness index is approximately constant up to a speed of 110 RPM, when the particles start being arranged in a spiral pattern on the bioreactor base and a drop of $I_{B}(N) / I_{B}(0)$ occurs. As the orbital speed is further increased a nearly constant value of the brightness index is attained above 150 RPM, implying that the "just-suspended" condition is achieved.

To better compare the results obtained for the different conditions analysed, the normalised brightness index, $I^{*}$, of Equation 5, which is scaled with the zero-speed, $I_{B}(0)$, and final-speed, $I_{B}(\infty)$, brightnesses, is used in the rest of the work.

$$
I^{*}=\frac{I_{B}(N)-I_{B}(\infty)}{I_{B}(0)-I_{B}(\infty)}
$$

The suspended speed is associated to a $95 \%$ decrease of the brightness index with respect to the zero-speed condition, and it is identified as the speed at which $I^{\star}=5 \%$. Based on the statistical error of the brightness index, $\approx 3 \%$, and the non-linear regression method used to fit the data points, the uncertainty affecting the just suspended speed was found to be $\approx 5 \%$.

A video showing the particle suspension dynamics is also provided in the supplementary materials (JS-Video.avi). In this case however the shaker table was started from still conditions and, similarly to standard operating procedures, was gradually accelerated to a final speed of $140 \mathrm{rpm}$ by the controller mounted on the shaker system (i.e. steady-state conditions were not achieved 
at intermediate speeds). As a consequence the instantaneous velocity associated to each frame is unknown, and the following discussion is made in terms of number of revolutions of the shaker tray (i.e. the encoder was used to acquire a frame per revolution). In agreement with the data reported in Figure 2, darker zones start appearing at the periphery of the bioreactor $(t=3-5$ $\mathrm{s}$ of the video), with microcarriers being more concentrated at the centre for increasing speed. This is well captured in Figure 3 (a), where the radial profiles of the normalised brightness index, $I^{*}(r)$, are shown for selected time instants, counted in number of revolutions, $n$, of the shaker tray, and corresponding to increasing shaking speed. After 100 revolutions, the shaker table has not gained a speed high enough to lift the particles, and the index $I^{*}$ is nearly constant across the bioreactor diameter and close to unity. As the shaker table is accelerated a drop of $I^{*}$ occurs after 110 revolutions, with the micriocarriers being suspended for $r / R \geq 0.6$, while the center of the bioreactor, $r / R \leq 0.3$, is still unaffected after 130 revolutions. It is worth noticing that also the rate of suspension is lower in proximity of the bioreactor axis. For example, a 10 revolutions increment $(n=120-130)$ for $r / R \geq 0.6$ determines a variation of the normalised brightness index of $\Delta I^{*} \approx 0.45$, while a similar $\operatorname{drop}(\approx 0.5)$ occurs at $r / R=0.3$ over a larger range of shaker revolutions, $\Delta n=30(n=140-170)$.

The spiral pattern, described in Figure 2 and shown in the supplementary video, is further analysed in Figure $3(\mathrm{~b})$, where the azimuthal profiles of $I^{*}$ are plotted at $r / R=0.8$ for an increasing number of shaker table revolutions $(n=100-135)$. It is evident that for $n=110-122$ the profiles show a cyclic variation in the azimuthal direction, with 5 peaks over the range of $\theta$ considered. As expected the intensity of the profiles is decreasing as more microcarriers are lifted with increasing speed (i.e. number of revolutions), and the profiles are randomly shifted with respect to each other along $\theta$, because the instants considered were taken far apart in time, and the spiral structure might have rotated with respect to the bioreactor. However an estimate of the spiral inclination can be gained from Figure 4 (a), where a single cycle of $I^{*}$ has been obtained through a phase-average, \langle\rangle , along the azimuthal direction with a period $\Delta \theta=20^{\circ}$. This analysis was performed at different radii for a single frame, $n=117$. The phase-averaged profiles were normalised by their maximum variation $\left\langle\Delta I_{B}\right\rangle$, so that the final brightness parameter assumed a maximum absolute intensity of $\approx 1$ for all the radii considered $(r / R=0.6-0.9)$. It should be noted that in Figure 4 (a) the flow direction is from right to left and opposite to that of $\theta$. The peak shifts to the right as the radius increases, which means that the spiral is oriented towards the center in the direction of motion. The variation of the peak azimuthal coordinate, $\theta_{\text {max }}$, against the radius is shown in Figure 4 (b) for two time instants, $n=117$ and 120 . The peak azimuthal coordinate, $\theta_{\max }$, shows a linear increase with $r / R$ and the slope magnitude is nearly the same for both instants considered (i.e. $18.57^{\circ}$ vs $18.86^{\circ}$ ). A visualisation of the spiral locus is provided in the inset diagram, where the arrow points in the flow direction.

The variation of $I^{*}$ against the shaker tray speed is plotted in Figures 5 (a) and (b) for two orbital diameters, $d_{o}=1.5$ and 2.5 , respectively. Three different microcarriers' concentrations are considered, $c=2.5,7.5$ and $12.5 \mathrm{~g} / \mathrm{L}$, while the fluid height and vessel size are kept constant 
( $h=5 \mathrm{~cm}, d_{i}=7 \mathrm{~cm}$ ). It should be noted that by definition the index, $I^{*}$, can assume only values between 0 and 1 at high and low shaking speeds, respectively. Data points are fitted with the model of equation 6 , where in the remainder part of the work the variable $x$ can either be the shaker speed, $N$, or the Froude number ratio, $F r / F r_{c r}$.

$$
I^{*}(x)=\frac{1}{1+\mathrm{e}^{a\left(x-x_{0}\right)}}
$$

The parameters $x_{0}$ and $a$ position the curve along the $x$ coordinate, and control its rate of decay, respectively. The plots of Figure 5 (a) cross the $5 \%$ reference line within a relative small range of suspension speeds, $N_{s}=153-160$ RPM, and a correlation between the concentration and the suspension speed seems to be present (i.e. lower suspension speeds occur for lower concentrations). However this correlation is not present in the data of Figure 5 (b) for $d_{o}=2.5 \mathrm{~cm}$, where an opposite behaviour is observed (i.e. lowest suspension speed for greatest concentration considered). Also in this case the range of variation of the suspended speed is relatively small, $N=145-152$ RPM, and it is within the error of the measurement technique employed. Based on this consideration it was concluded that the concentration should not affect to a large extent the suspension of the microcarriers, at least within the range of concentration considered in this study, which includes those commonly employed in the bioprocess industry.

On the contrary the variation of the suspension speed with the orbital diameter is significant. This is evident in Figure 6 (a) where the normalised brightness index, $I^{*}$, is plotted against the shaker speed for different orbital diameter, $d_{o}=1.5,2.5$ and $5 \mathrm{~cm}$. As expected the suspension speed, $N_{s}$, increases with decreasing orbital diameter, and assumes values of 120 RPM, 144 RPM and $153 \mathrm{RPM}$ for $d_{o}=5 \mathrm{~cm} 2.5 \mathrm{~cm}$ and $1.5 \mathrm{~cm}$, respectively. In Figure 6 (b) an attempt was made to assess whether the suspension mechanism would scale with the critical Froude number ratio, $F r / F r_{c r}$. In fact the three systems are associated to different $d_{o} / d_{i}$ and therefore reach the flow transition at different speeds (Weheliye et al., 2013). However the plot of Figure 6 (b) does not support this scaling procedure with the lowest (highest) orbital diameter still being associated to the greatest (lowest) critical Froude number ratio. This was explained by considering that the fluid height $(h=5 \mathrm{~cm})$ of two, $d_{o}=1.5 \mathrm{~cm}$ and $2.5 \mathrm{~cm}$, out of the three systems investigated is too large for the flow to fully develop to the cylinder bottom before transition occurs. In both cases $h / d_{i}>\sqrt{d_{o} / d_{i}}\left(0.71>0.46\right.$ for $d_{o}=1.5 \mathrm{~cm}$ and $0.71>0.59$ for $d_{o}=2.5$ $\mathrm{cm}$ ) and Equation 2 shall be used to determine the critical Froude number, $F r_{c r}$.

Based on these considerations a second set of measurements was carried out to assess the suspension process when $h / d_{i} \leq \sqrt{d_{o} / d_{i}}$, and a critical speed exists for the flow to extend to the bottom of the reactor. The variation of $I^{*}$ with $d_{o}$ is provided in Figures 7 (a) and (b) for increasing speed and critical Froude number ratio, respectively. In agreement with Figure 6 (a) the plots of Figure 7 (a) intercept the $5 \%$ reference line at increasing suspension speed for decreasing orbital diameter. In this case however when the brightness index is plotted against the critical Froude number ratio (see Figure $7 \mathrm{~b}$ ) the data tend to collapse on a single curve, indicating that the parameter $F r / F r_{c r}$ can be successfully used for scaling across different configurations (i.e. $d_{o} / d_{i}$ ), provided that the fluid height satisfies the condition $h / d_{i} \leq \sqrt{d_{o} / d_{i}}$. 
The data presented in Figures 6 and 7 are summarised in Figure 8, where the suspended to critical Froude number ratio is plotted against the parameter $h / d_{i} / \sqrt{d_{o} / d_{i}}$. As indicated by the inset schematics values of $h / d_{i} / \sqrt{d_{o} / d_{i}}<1$ identify those configurations for which the toroidal vortices extend to the bottom of the bioreactor when the critical speed is achieved, while this does not occur for $h / d_{i} / \sqrt{d_{o} / d_{i}}>1$, and flow transition takes place without the flow developing to the reactor base. The error bars in Figure 8 are supposed to provide a reference, and correspond to a $2 \mathrm{RPM}$ variation in the suspension speed $N_{s}$ (i.e. $\left.\mathrm{d} F r_{s} / F r_{c r}=2 \times\left(N_{s} / N_{c r}^{2}\right) \mathrm{d} N_{s}\right)$. From Figure 8, the $95 \%$ suspension condition is achieved for $F r_{s} / F r_{c r} \leq 1.1$ when $h / d_{i} / \sqrt{d_{o} / d_{i}}<1$, while the suspended to critical Froude number ratio tends to drift further away from the dashed reference line at $F r_{s} / F r_{c r}=1.1$ as $h / d_{i} / \sqrt{d_{o} / d_{i}}$ increases above 1. It is interesting to note that the suspension speed data obtained by Olmos et al. (2015) in Erlenmeyer flasks showed a good scaling with the critical speed, $N_{c r}$, also for $h / d_{i} / \sqrt{d_{o} / d_{i}}>1$.

The coefficients $a$ and $x_{0}$ of Equation 6, used to determine the suspended to critical Froude number ratio (i.e. $F r_{s} / F r_{c r}=\log (19) / a+x_{0}$ for $95 \%$ suspension), are provided in Table 2. It is worth pointing that the range of variation of the decay coefficient for data associated to $h / d_{i} / \sqrt{d_{o} / d_{i}}>1(7<a<14.3)$ is lower than that for $h / d_{i} / \sqrt{d_{o} / d_{i}}<1(14<a<17.8)$. This implies that for $h / d_{i} / \sqrt{d_{o} / d_{i}}<1$ suspension occurs more sharply with increasing speed.

\begin{tabular}{|c|c|c|c|c|c|c|c|c|c|c|}
\hline \multicolumn{8}{|c|}{$h=5 \mathrm{~cm}$} & \multicolumn{3}{|c|}{$h=3 \mathrm{~cm}$} \\
\hline \multicolumn{3}{|c|}{$d_{o}=1.5 \mathrm{~cm}$} & \multicolumn{3}{|c|}{$d_{o}=2.5 \mathrm{~cm}$} & \multicolumn{2}{|c|}{$d_{o}=5 \mathrm{~cm}$} & $2 \mathrm{~cm}$ & $3 \mathrm{~cm}$ & $4 \mathrm{~cm}$ \\
\hline $2.5 \mathrm{~g} / \mathrm{L}$ & $7.5 \mathrm{~g} / \mathrm{L}$ & $12.5 \mathrm{~g} / \mathrm{L}$ & $2.5 \mathrm{~g} / \mathrm{L}$ & $7.5 \mathrm{~g} / \mathrm{L}$ & $12.5 \mathrm{~g} / \mathrm{L}$ & $7.5 \mathrm{~g} / \mathrm{L}$ & $12.5 \mathrm{~g} / \mathrm{L}$ & \multicolumn{3}{|c|}{$c=2.5 \mathrm{~g} / \mathrm{L}$} \\
\hline 13.1 & 9.8 & 12.8 & 7.1 & 14.3 & 10 & 14 & 14.13 & 17.57 & 15.39 & 17.83 \\
\hline 1.05 & 1.05 & 1.19 & 0.87 & 0.98 & 0.87 & 0.72 & 0.74 & 0.95 & 0.92 & 0.94 \\
\hline \multicolumn{6}{|c|}{$h / d_{i} / \sqrt{d_{o} / d_{i}}>1$} & \multicolumn{5}{|c|}{$h / d_{i} / \sqrt{d_{o} / d_{i}}<1$} \\
\hline
\end{tabular}

Table 2: Coefficients $a$ and $x_{0}$ obtained for all the sets of data analysed in this work.

\subsection{Microcarriers' dispersion}

A similar analysis to that employed in the previous section was carried out over vertical planes of measurement to assess the dispersion across the bioreactor of the microcarriers' suspension. In this case the normalisation of the brightness index was done according to Equation 7, where the coefficient varies from 0 (low concentration of microcarriers' over the volume) to 1 (homogenous concentration across the bioreactor volume).

$$
I^{*}=\frac{I_{B}(N)-I_{B}(0)}{I_{B}(\infty)-I_{B}(0)}
$$

The variation of $I^{*}$ with the critical Froude number ratio, $F r / F r_{c r}$, is provided in Figure 9, where inset snapshots provide a visual reference of the degree of dispersion. Data refer to a 
system with $d_{i}=13 \mathrm{~cm}, d_{o}=5 \mathrm{~cm}$ and $h=6.5 \mathrm{~cm}\left(h / d_{i} / \sqrt{d_{o} / d_{i}}<1\right)$. The vertical and horizontal lines provide a reference of the suspended to critical Froude number ratio, $F r_{s} / F r_{c r}=1.1$, found in the previous section, and of the $95 \%$ degree of homogeneity, respectively. From Figure 9 it can be concluded that complete dispersion is achieved at a speed slightly higher than the suspended one, $\approx 1.2 \times F r_{c r}(95 \%$ threshold $)$.

A closer view at the dispersion of microcarriers across the tank can be gained from the axial and radial cumulative brightness profiles of Figures 10 (a) and (b), respectively $\left(d_{i}=10 \mathrm{~cm}\right.$, $d_{o}=h=5 \mathrm{~cm}$ ). The axial (radial) cumulative brightness was obtained by adding the image brightness along the radial (axial) direction. Before proceeding with the discussion, it is worth mentioning that a limitation of adopting the brighness index as a reference for microcarriers' concentration is that in the vertical plane of measurements the laser enters the bioreactor from the base, and therefore complete brightness homogeneity is impossible to achieve due to reflections. This explains why brightness maxima are always located at $z=0$, even at the higher speed investigated, when microcarriers' suspension has certainly occurred. Despite this the current data provide a reliable description of the suspension over a vertical plane for increasing speed. Bearing this in mind, the plot of Figure 10 (a) shows that the axial distribution of microcarriers is poor for $N \leq 100$ with the normalised brightness index, $I_{B}(z, N) / I_{B}(0, N)$, being relatively low for $z / d_{i} \leq 0.04$, while, in agreement with the higher decay coefficients observed in Table 2 for $h / d_{i} / \sqrt{d_{o} / d_{i}}<1$, a sharp change in $I_{B}(z, N) / I_{B}(0, N)$ occurs over a relatively small range of shaker speeds, $N=100-105 \mathrm{RPM}$. The curves of $N=105 \mathrm{RPM}$ and $N=130 \mathrm{RPM}$ are nearly parallel for $z / d_{i} \geq 0.06$ indicating that a similar degree of dispersion along the axial direction has been achieved for both, while the lower intensity of $I_{B}(z, N) / I_{B}(0, N)$ indicates that fewer microcarriers are suspended for the lower speed considered.

Similarly to the axial profiles, the radial profiles of the cumulative brightness index, $\left(I_{B}(z, N)-\right.$ $\left.I_{B}(0, N)\right) / I_{B}(0, N)$, Figure 10 (b), show little suspension for $N<102$, while at greater speeds the radial distribution is characterised by double crested profiles, where the peaks capture the higher microcarriers' concentration already present in the top-right inset of Figure 9. The peaks are located close to the reactor axis and they occur in the region swept by the precessional vortex once flow transition has occurred. Based on these results and those in the previous section it can be concluded that microcarriers are pushed from the periphery towards the centre of the reactor base, and they are then sucked into the bulk flow by the depression created close to the axis of the bioreactor by the two-counter rotating and precessional vortices, before and after flow transition, respectively.

\subsection{Two-phase flow dynamics}

Two-phase Particle Image Velocimetry experiments were carried out to better understand the influence of the solid phase on the mean characteristics of the flow, and to assess whether the flow transition reported by Weheliye et al. (2013) can be extended to the two-phase system. A preliminary analysis was carried out to assess whether the free surface wave, which is the flow 
driving mechanism, is affected by the microcarriers' concentration. The study of Weheliye et al. (2013) showed that for a single-phase system the nondimensional wave amplitude, $\Delta h / d_{i}$, is proportional to the Froude number, meaning that for selected combinations of $N$ and $d_{o}$, the free surface will assume a fixed inclination, which is independent of the fluid height $h$ and vessel diameter, $d_{i}$. The constant of proportionality, $a_{o}$, depends on the fluid considered, and is equal to 1.4 in the case of water, and decreases with increasing fluid viscosity (Ducci and Weheliye, 2014). The variation of $\Delta h / d_{i}$ against $\operatorname{Fr}(0.25<F r<0.5)$ for different microcarriers' concentrations at $h / d_{i}=0.5$, and $d_{o} / d_{i}=0.5$ is provided in Figure 11. The data points are all located close to the reference line, which corresponds to a single-phase system with water as the working fluid $\left(a_{o w}=1.4\right)$. A small decrease of the slope might be seen for increasing microcarriers' concentrations, that is consistent with the behaviour reported by Ducci and Weheliye (2014) for increasing viscosity. This means that the flow dynamics of the two-phase system is not remarkably affected by the presence of microcarriers at the concentration considered, and that the applicability of the relation found by Weheliye et al. (2013) can be extended to the two-phase system. Lower values of the slope coefficient, $a_{o}$, might imply that the critical Froude number for the two-phase system is slightly higher than that of the single-phase (see Equation 1), and therefore the suspended speed data points of Figure 8 might get closer to the horizontal reference line of $\mathrm{Fr} / \mathrm{Fr}_{\mathrm{cr}}=1$.

The phase-resolved velocity vector fields and tangential vorticity, $\omega_{\theta} /(\pi N)$, contour maps of the liquid and solid phases are shown in Figure 12 (a-b) and (c-d) for in-phase, prior to flow transition, and out-of-phase conditions, respectively. For both flow conditions the phase angle was $\phi=0$ and the microcarriers' concentration, $c=0.5 \mathrm{~g} / \mathrm{L}$. The velocity fields of the liquid and solid phases for in-phase flow (Figures $12 \mathrm{a}$ and b) are qualitatively similar to each other, and are characterised by the two vortical cell configuration already identified by Weheliye et al. (2013) at the same speed for single-phase flow. However, in the toroidal vortex region, the vorticity of the solid phase assumes values slightly higher than for the liquid one (mainly on the left hand side vortex), indicating that a slip velocity is present between the two phases. Similar conclusions can be drawn when comparing the velocity fields for the out of phase flow (Figures $12 \mathrm{c}$ and $\mathrm{d})$. In this case the axial slip velocity, $\left|u_{z_{S}}-u_{z_{L}}\right|<0.02 \times \pi N d_{o}(0-6 \mathrm{~mm} / \mathrm{s})$. It is worth mentioning that this range of values is comparable to the average and maximum velocities of the liquid phase over the plane of measurement, 0.033 and $0.10 \times \pi N d_{o}$, respectively.

\section{Conclusions}

This study is the first one to provide insight on the two-phase flow dynamics occurring in an orbitally shaken bioreactor when microcarriers are used in suspension under real process conditions. The suspension dynamics of the two-phase system was investigated using a visualization approach, which allowed to estimate the "just - suspended" shaking speed from the light scattered by the microcarriers on a laser plane parallel to the bottom of the cylindrical bioreactor. The shaking system was studied varying solid concentration and orbital diameter, and the results highlightened the correlation between the microcarriers suspension and the critical Froude 
number corresponding to the occurrence of the flow transition identified by Weheliye et al. (2013) for a single-phase system. It was found that for bioreactor configurations corresponding to $h / d_{i} / \sqrt{d_{o} / d_{i}}<1$ the suspended Froude number, $F r_{s}$, is nearly constant and equal to $1.1 \times F r_{c r}$, while for $h / d_{i} / \sqrt{d_{o} / d_{i}}>1$ the suspended speed tends to increase, and suspension is delayed to higher speeds after flow transition. From this point of view the first type of configuration should be sought because it achieves full suspension and at the same time minimises power consumption and shear rates.

An analysis of the suspension mechanisms highlighted that microcarriers are pushed from the perisphery towards the centre of the reactor base along a spiral pattern, and then they are sucked into the bulk flow by the depression created close to the axis of the bioreactor by the two-counter rotating and precessional vortices, before and after flow transition, respectively. Vertical plane measurements were used to assess the homogeneity of the microcarriers across the reactor volume, and it was found that full dispersion is achieved at $\approx 1.2 \times F r_{c r}$. A model was developed to fit the suspension data, and showed that suspension dynamics are faster and occur over a narrower range of speeds for $h / d_{i} / \sqrt{d_{o} / d_{i}}<1$. The free surface experiments validated the relation found by Weheliye et al. (2013) between the non-dimensional wave amplitude of the cylindrical bioreactor, $\Delta h / d_{i}$, and the Froude number, and it was found that the presence of the microcarriers might reduce the constant of proportionality between the two parameters, and result in slightly higher critical Froude number, $F r_{c r}$. The velocity fields of the liquid and solid phases were simultaneously measured over a vertical plane bisecting the vessel, and their mean flows were found to be very similar both for in-phase and out-of-phase conditions. This is in agreement with previous studies on stirred tank reactors where low solid concentrations are employed. The range of variation of the axial slip velocity, $\left|u_{z_{S}}-u_{z_{L}}\right|<0.02 \times \pi N d_{o}(0-6$ $\mathrm{mm} / \mathrm{s}$ ), was comparable in magnitude to the average and maximum velocities of the liquid phase over the plane of measurement, 0.033 and $0.10 \times \pi N d_{o}$, respectively.

Further studies are called for to investigate the suspension dynamics of the next generation of microcarriers. Biodegradable materials are increasingly used to make microcarriers for cell adherent applications in order to avoid the need for the cell detachment and recovery steps. However the materials used are often characterised by densities much heavier than water, thus requiring considerable energy to be suspended. The flow visualisation methodology established in this work, as well as the simultaneous measurement of the two-phase flow characteristics, could be implemented for other microcarriers' types to assess the quality of suspension, and its dependence on the bioreactor geometry and operating conditions. 


\section{Nomenclature}

\section{Abbreviation}

2D Two-Dimensional

OSB Orbitally shaken bioreactor

STR Stirred Tank Reactor

PIV Particle Image Velocimetry

3D Three-Dimensional

\section{Greek Symbols}

$\nu \quad$ Kinematic viscosity, $\mathrm{m}^{2} / \mathrm{s}$

$\rho \quad$ Microcarriers' density $\mathrm{kg} / \mathrm{m}^{3}$

$\rho^{\star} \quad$ Microcarriers' relative density, -

$\phi \quad$ Phase angle of the table, ${ }^{\circ}$

$\omega_{i} \quad$ Vorticity component in the $i$ th direction, $\mathrm{s}^{-1}$

\section{Roman Symbols}

a Decay coefficient of Equation 6,-

$a_{\text {ow }} \quad$ Constant of proportionality for water, -

$d_{i} \quad$ Inner diameter of the cylinder, $\mathrm{m}$

$d_{o} \quad$ Orbital diameter, $\mathrm{m}$

$d_{p}, d_{50}$ Microcarriers' diameter, $\mathrm{m}$

$\mathrm{Fr} \quad$ Froude number, -

$F r_{c r}$ Critical/transitional Froude number, -

$\mathrm{Fr}_{s} \quad$ Suspended Froude number, -

$g \quad$ Gravitational acceleration, $\mathrm{m} / \mathrm{s}^{2}$

$h \quad$ Fluid height at rest, $m$

$\Delta h \quad$ Free surface height, $\mathrm{m}$

$I^{*} \quad$ Normalised brightness index, -

$I_{B} \quad$ Brightness index, -

$n \quad$ Number of shaker revolution, -

$N \quad$ Shaking frequency, $\mathrm{s}^{-1}$

$N_{c r} \quad$ Critical shaking frequency, $\mathrm{s}^{-1}$

$N_{s} \quad$ Suspension shaking frequency, $\mathrm{s}^{-1}$

$R \quad$ Inner radius of the cylinder, $\mathrm{m}$

Re Reynolds number, -

$u_{i} \quad$ Velocity in the $i$ th direction, $\mathrm{m} / \mathrm{s}$

$V_{f} \quad$ Fluid filling volume, $\mathrm{m}^{3}$

$x_{0} \quad$ Position coefficient of Equation 6, - 


\section{List of Figures}

1 Experimental set-ups: (a) suspended speed; (b) two-phase PIV. . . . . . . . . . . 19

2 Visualization of the suspension mechanism and variation of the brightness percentage index, $I_{B}(N) / I_{B}(0)$, with shaking speed $\left(d_{o}=1.5 \mathrm{~cm}, h=5 \mathrm{~cm}, c=2.5\right.$

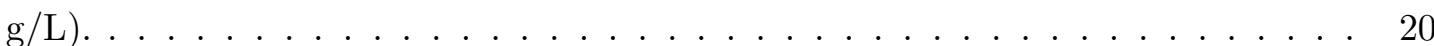

3 Profiles of the normalised brightness index $I^{*}$ for increasing number of shaker revolutions $\left(d_{o}=2 \mathrm{~cm}, h=3 \mathrm{~cm}, c=2.5 \mathrm{~g} / \mathrm{L}\right.$ ): (a) radial profiles; (b) azimuthal profiles $(r / R=0.8) \ldots \ldots \ldots \ldots \ldots \ldots$

4 (a) Phase-averaged azimuthal profiles of the image brightness at $n=117$ for different radii $(r / R=0.6-0.9)$; (b) Radial and azimuthal coordinates of the brightness peak for $n=117$ and $120\left(d_{o}=2 \mathrm{~cm}, h=3 \mathrm{~cm}, c=2.5 \mathrm{~g} / \mathrm{L}\right) \ldots \ldots$

$5 \quad$ Variation of $I^{*}$ with shaker speed for different microcarriers' concentrations ( $h=5$ $\mathrm{cm}, d_{i}=7 \mathrm{~cm}$ ): (a) $d_{o}=1.5 \mathrm{~cm}$; (b) $d_{o}=2.5 \mathrm{~cm} \ldots \ldots \ldots 23$

6 Variation of $I^{*}$ for different orbital diameters $\left(h=5 \mathrm{~cm}, d_{i}=7 \mathrm{~cm}, c=2.5 \mathrm{~g} / \mathrm{L}\right)$ : (a) variation with shaker speed, $N$; (b) variation with $F r / F r_{c r} \ldots \ldots \ldots$. . . . 24

$7 \quad$ Variation of $I^{*}$ for different orbital diameters $\left(h=3\right.$ and $5 \mathrm{~cm}, d_{i}=7 \mathrm{~cm}, c=2.5$ g/L): (a) variation with shaker speed, $N$; (b) variation with $F r / F r_{c r}$. . . . . .

8 Variation of the suspended to critical Froude number ratio, $F r_{s} / F r_{c r}$, with critical height ratio, $\frac{h}{d_{i}} / \sqrt{\frac{d_{o}}{d_{i}}}$, for all the conditions investigated. . . . . . . . . . 26

9 Variation of the normalised brightness index, $I^{*}$, with the Froude number ratio, $F r / F r_{c r}$, obtained from measurements on a vertical plane $\left(d_{i}=13 \mathrm{~cm}, d_{o}=5 \mathrm{~cm}\right.$,

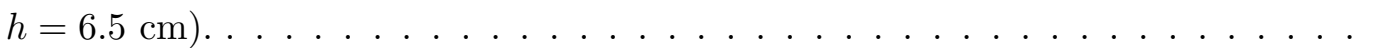

10 Profiles of the axial and radial cumulative brightness indices for increasing shaken speed $\left(d_{i}=10 \mathrm{~cm}, d_{o}=5 \mathrm{~cm}, h=5 \mathrm{~cm}\right.$ ): (a) Axial profiles; (b) Radial profiles. .

11 (a) Variation of the non-dimensional wave amplitude, $\Delta h / d_{i}$, with Froude number, $F r$, for different microcarriers' concentrations $\left(h=5 \mathrm{~cm}, d_{i}=10 \mathrm{~cm}, d_{o}=5\right.$

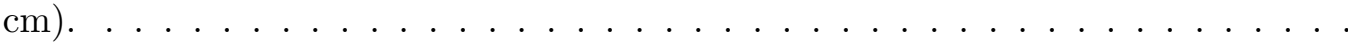

12 (a) Velocity vector fields and tangential vorticity contour maps of the liquid and solid phases before and after flow transition $\left(h=5 \mathrm{~cm}, d_{i}=10 \mathrm{~cm}, d_{o}=5 \mathrm{~cm}\right.$, $c=0.5 \mathrm{~g} / \mathrm{L}$ ): (a) Liquid phase, $N=90 \mathrm{RPM}$; (b) Solid phase, $N=90 \mathrm{RPM}$; (c) Liquid phase, $N=110$ RPM; (d) Solid phase, $N=110$ RPM . . . . . . . . . 


\section{References}

Collignon, M.L.L., Delafosse, A., Crine, M., Toye, D., 2010. Axial impeller selection for anchorage dependent animal cell culture in stirred bioreactors: Methodology based on the impeller comparison at just-suspended speed of rotation. Chemical Engineering Science 65, 5929-5941.

Discacciati, M., Hacker, D., Quarteroni, A., Quinodoz, S., Tissot, S., Wurm, F.M., 2012. Numerical simulation of orbitally shaken viscous fluids with free surface. International Journal for Numerical Methods in Fluids, 1-14.

Ducci, A., Weheliye, W.H., 2014. Orbitally shaken bioreactors - Viscosity effects on flow characteristics. AIChE Journal 60, 3951-3968.

Ferrari, C., Balandras, F., Guedon, E., Olmos, E., Chevalot, I., Marc, A., 2012. Limiting cell aggregation during mesenchymal stem cell expansion on microcarriers. Biotechnology Progress $28,780-787$.

Frauenschuh, S., Reichmann, E., Ibold, Y., Goetz, P.M., Sittinger, M., Ringe, J., 2007. A microcarrier-based cultivation system for expansion of primary mesenchymal stem cells. Biotechnology Progress 23, 187-193.

GE Healthcare Life Sciences, 2013. Microcarrier Cell Culture-Principles \& Methods. Technical Report.

Gomez, C., Bennington, C.P.J., Taghipour, F., 2010. Investigation of the Flow Field in a Rectangular Vessel Equipped With a Side-Entering Agitator. Journal of Fluids Engineering $132,051106$.

Ismadi, M.Z., Gupta, P., Fouras, A., Verma, P., Jadhav, S., Bellare, J., Hourigan, K., 2014. Flow characterization of a spinner flask for induced pluripotent stem cell culture application. PloS one 9, e106493.

Kim, H.M., Kizito, J.P., 2009. Stirring Free Surface Flows Due To Horizontal Circulatory Oscillation of a Partially Filled Container. Chemical Engineering Communications 196, 13001321.

King, J.A., Miller, W.M., 2007. Bioreactor development for stem cell expansion and controlled differentiation. Current Opinion in Chemical Biology 11, 394-398.

Lara, A.R., Galindo, E., Ramírez, O.T., Palomares, L.A., 2006. Living with heterogeneities in bioreactors: understanding the effects of environmental gradients on cells. Molecular biotechnology 34, 355-381.

Liu, N., Zang, R., Yang, S.T., Li, Y., 2014. Stem cell engineering in bioreactors for large-scale bioprocessing. Engineering in Life Sciences 14, 4-15. 
Mancilla, E., Palacios-Morales, C.A., Córdova-Aguilar, M.S., Trujillo-Roldán, M.A., Ascanio, G., Zenit, R., 2015. A hydrodynamic description of the flow behavior in shaken flasks. Biochemical Engineering Journal 99, 61-66.

Mohamet, L., Lea, M.L., Ward, C.M., 2010. Abrogation of E-cadherin-mediated cellular aggregation allows proliferation of pluripotent mouse embryonic stem cells in shake flask bioreactors. PloS one 5, e12921.

Nienow, A.W., Rafiq, Q.A., Coopman, K., Hewitt, C.J., 2014. A potentially scalable method for the harvesting of hMSCs from microcarriers. Biochemical Engineering Journal 85, 79-88.

Olmos, E., Loubiere, K., Martin, C., Delaplace, G., Marc, A., 2015. Critical agitation for microcarrier suspension in orbital shaken bioreactors: Experimental study and dimensional analysis. Chemical Engineering Science 122, 545-554.

Reclari, M., Dreyer, M., Tissot, S., Obreschkow, D., Wurm, F.M., Farhat, M., 2014. Surface wave dynamics in orbital shaken cylindrical containers. Physics of Fluids 26.

Rodriguez, G., Anderlei, T., Micheletti, M., Yianneskis, M., Ducci, A., 2014. On the measurement and scaling of mixing time in orbitally shaken bioreactors. Biochemical Engineering Journal 82, 10-21.

Rodriguez, G., Weheliye, W., Anderlei, T., Micheletti, M., Yianneskis, M., Ducci, A., 2013. Mixing time and kinetic energy measurements in a shaken cylindrical bioreactor. Chemical Engineering Research and Design 91, 2084-2097.

Sart, S., Schneider, Y.J., Agathos, S.N., 2009. Ear mesenchymal stem cells: an efficient adult multipotent cell population fit for rapid and scalable expansion. Journal of biotechnology 139, 291-299.

Schop, D., Janssen, F.W., Borgart, E., de Bruijn, J.D., van Dijkhuizen-Radersma, R., 2008. Expansion of mesenchymal stem cells using a microcarrier-based cultivation system: growth and metabolism. Journal of tissue engineering and regenerative medicine 2, 126-135.

Schop, D., Janssen, F.W., van Rijn, L.D.S., Fernandes, H., Bloem, R.M., de Bruijn, J.D., van Dijkhuizen-Radersma, R., 2009. Growth, metabolism, and growth inhibitors of mesenchymal stem cells. Tissue Engineering - Part A 15.

Simaria, A.S., Hassan, S., Varadaraju, H., Rowley, J., Warren, K., Vanek, P., Farid, S.S., 2014. Allogeneic cell therapy bioprocess economics and optimization: single-use cell expansion technologies. Biotechnology and bioengineering 111, 69-83.

Storm, M.P., Orchard, C.B., Bone, H.K., Chaudhuri, J.B., Welham, M.J., 2010. Threedimensional culture systems for the expansion of pluripotent embryonic stem cells. Biotechnology and bioengineering 107, 683-695. 
Tissot, S., Farhat, M., Hacker, D.L., Anderlei, T., Kühner, M., Comninellis, C., Wurm, F., 2010. Determination of a scale-up factor from mixing time studies in orbitally shaken bioreactors. Biochem. Eng. J. 52, 181-186.

Weheliye, W., Yianneskis, M., Ducci, A., 2013. On the Fluid Dynamics of Shaken Bioreactors - Flow Characterization and Transition. AIChE Journal 59, 334-344.

Zhang, H., Lamping, S.R., Pickering, S.C.R., Lye, G.J., Shamlou, P.A., 2008. Engineering characterisation of a single well from 24-well and 96-well microtitre plates. Biochem. Eng. J. 40, 138-149.

Zhang, H., Williams-Dalson, W., Keshavarz-Moore, E., Shamlou, P.A., 2005. Computationalfluid-dynamics (CFD) analysis of mixing and gas-liquid mass transfer in shake flasks. Biotechnology and applied biochemistry 41, 1-8.

Zhang, X., Bürki, C.A.A., Stettler, M., De Sanctis, D., Perrone, M., Discacciati, M., Parolini, N., DeJesus, M., Hacker, D.L., Quarteroni, A., Wurm, F.M., 2009. Efficient oxygen transfer by surface aeration in shaken cylindrical containers for mammalian cell cultivation at volumetric scales up to 1000L. Biochem. Eng. J. 45, 41-47. 


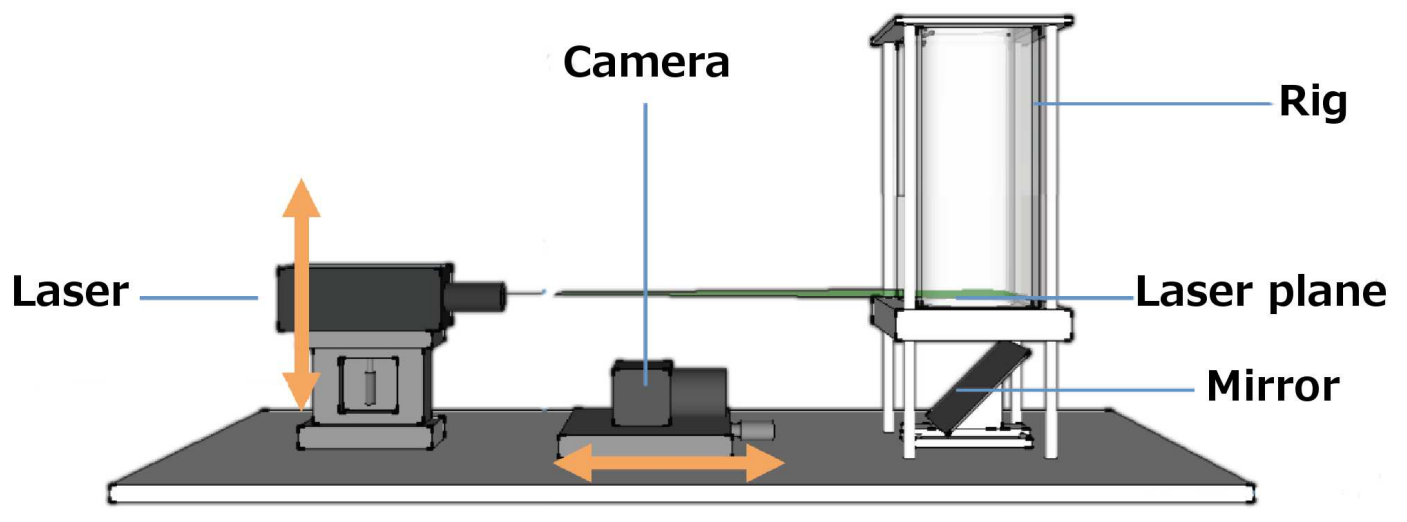

(a)

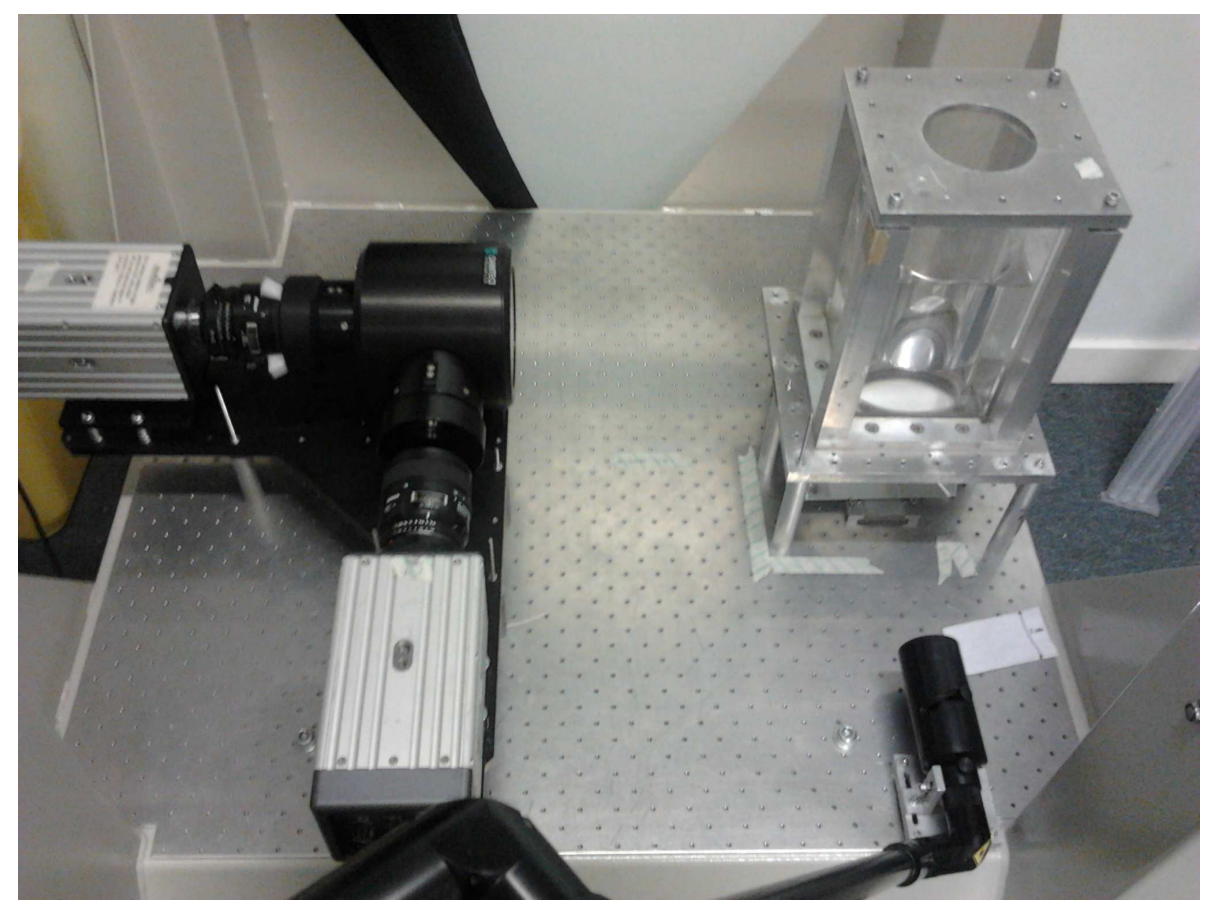

(b)

Figure 1: Experimental set-ups: (a) suspended speed; (b) two-phase PIV. 


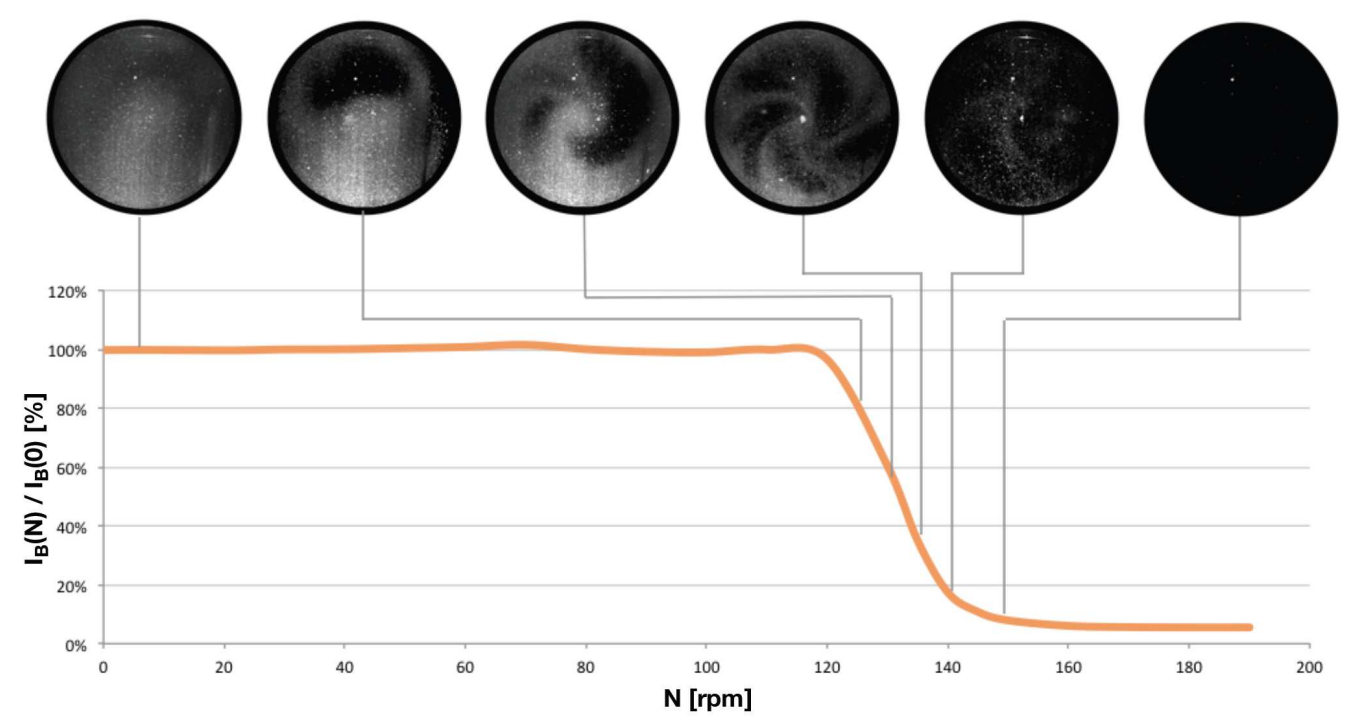

Figure 2: Visualization of the suspension mechanism and variation of the brightness percentage index, $I_{B}(N) / I_{B}(0)$, with shaking speed $\left(d_{o}=1.5 \mathrm{~cm}, h=5 \mathrm{~cm}, c=2.5 \mathrm{~g} / \mathrm{L}\right)$. 


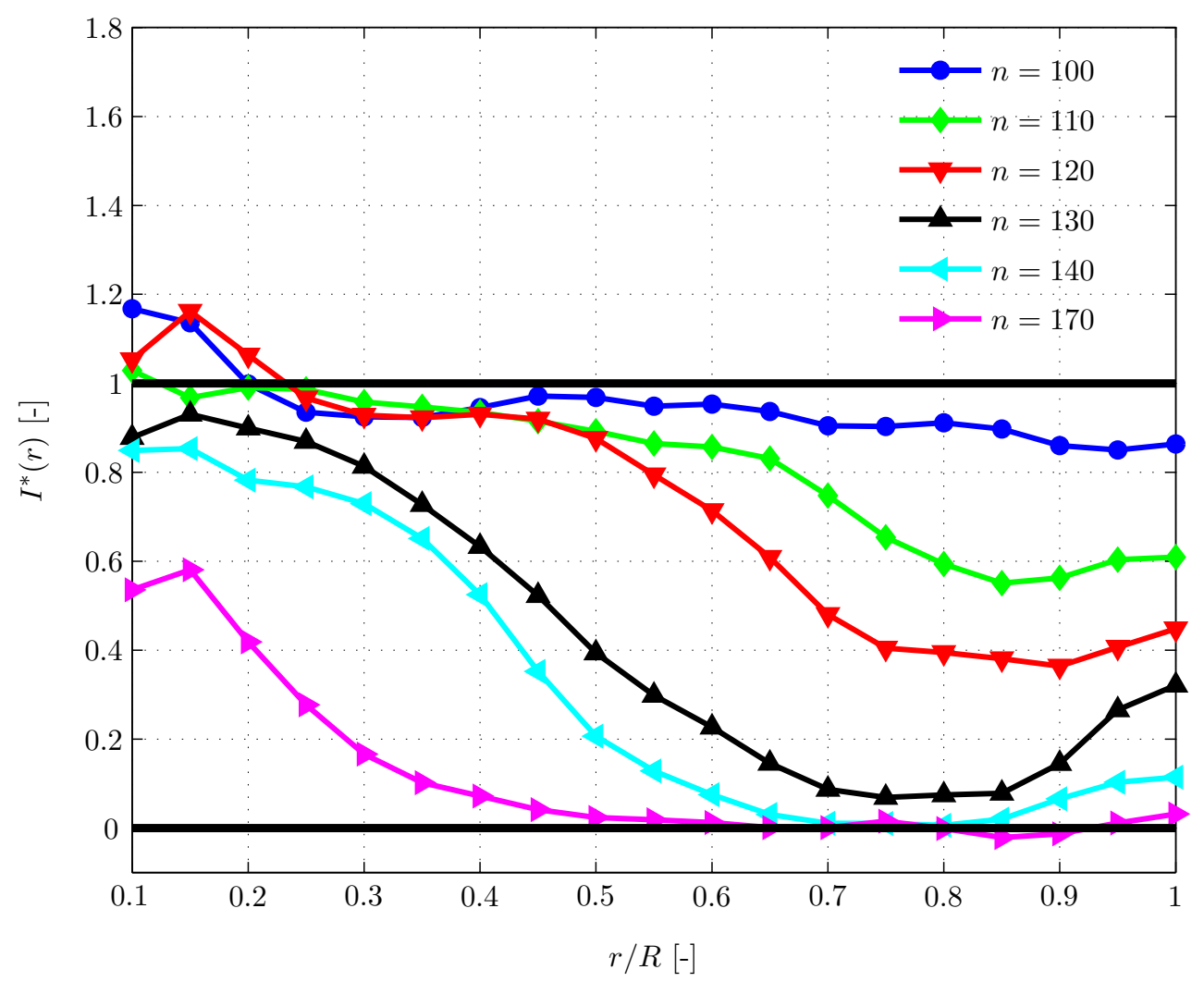

(a)

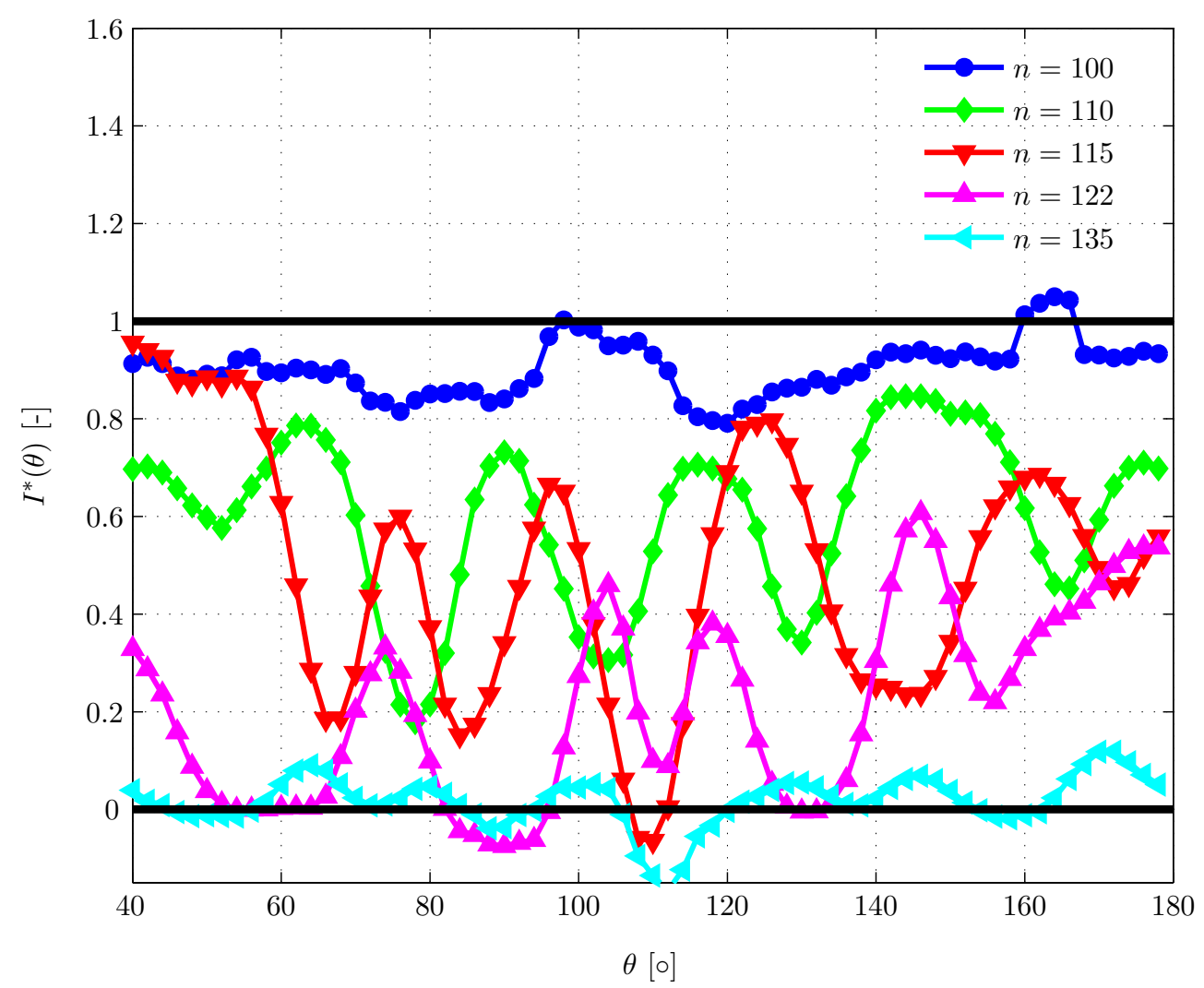

(b)

Figure 3: Profiles of the normalised brightness index $I^{*}$ for increasing number of shaker revolutions $\left(d_{o}=2 \mathrm{~cm}\right.$, $h=3 \mathrm{~cm}, c=2.5 \mathrm{~g} / \mathrm{L}$ ): (a) radial profiles; (b) azimuthal profiles $(r / R=0.8)$. 


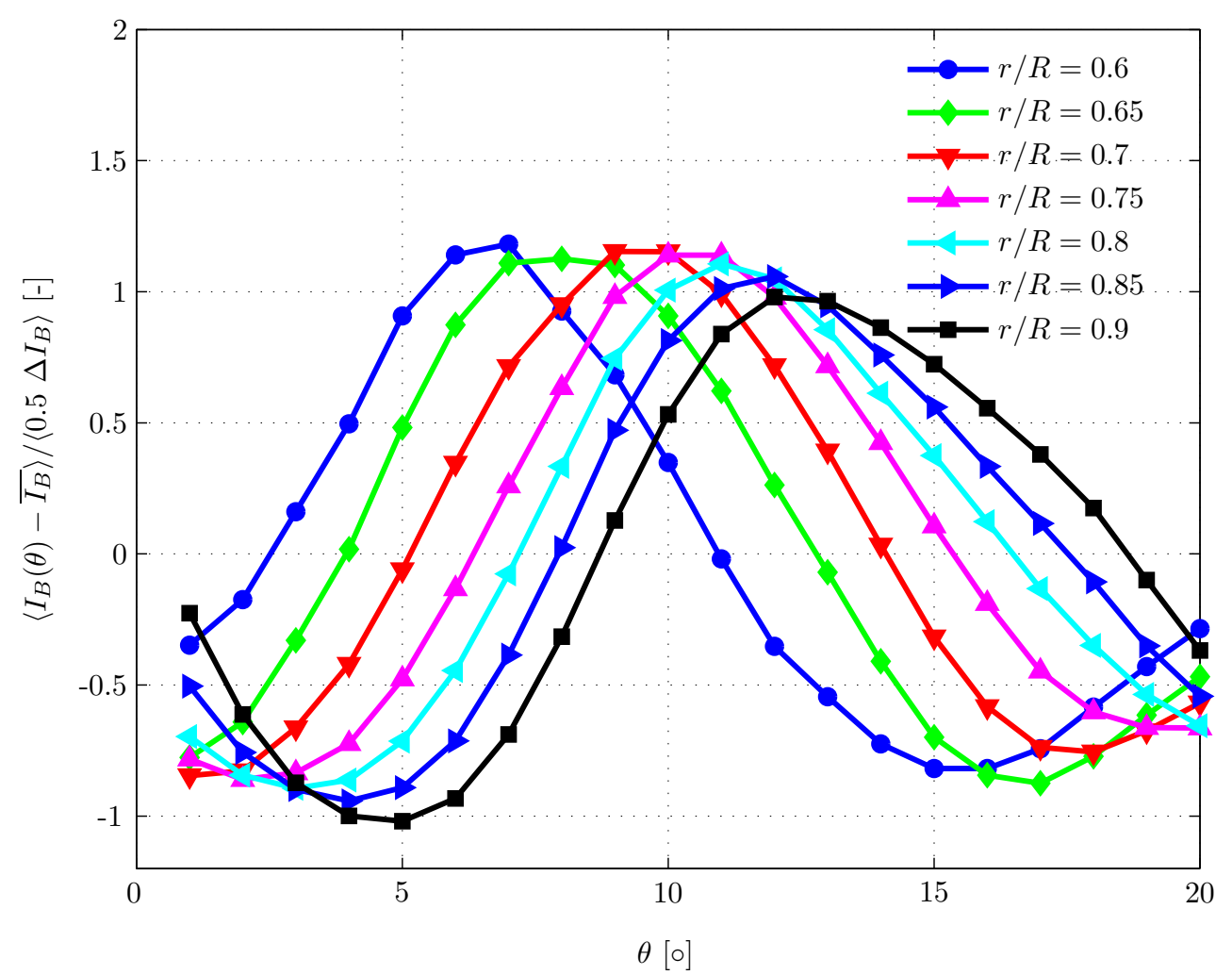

(a)

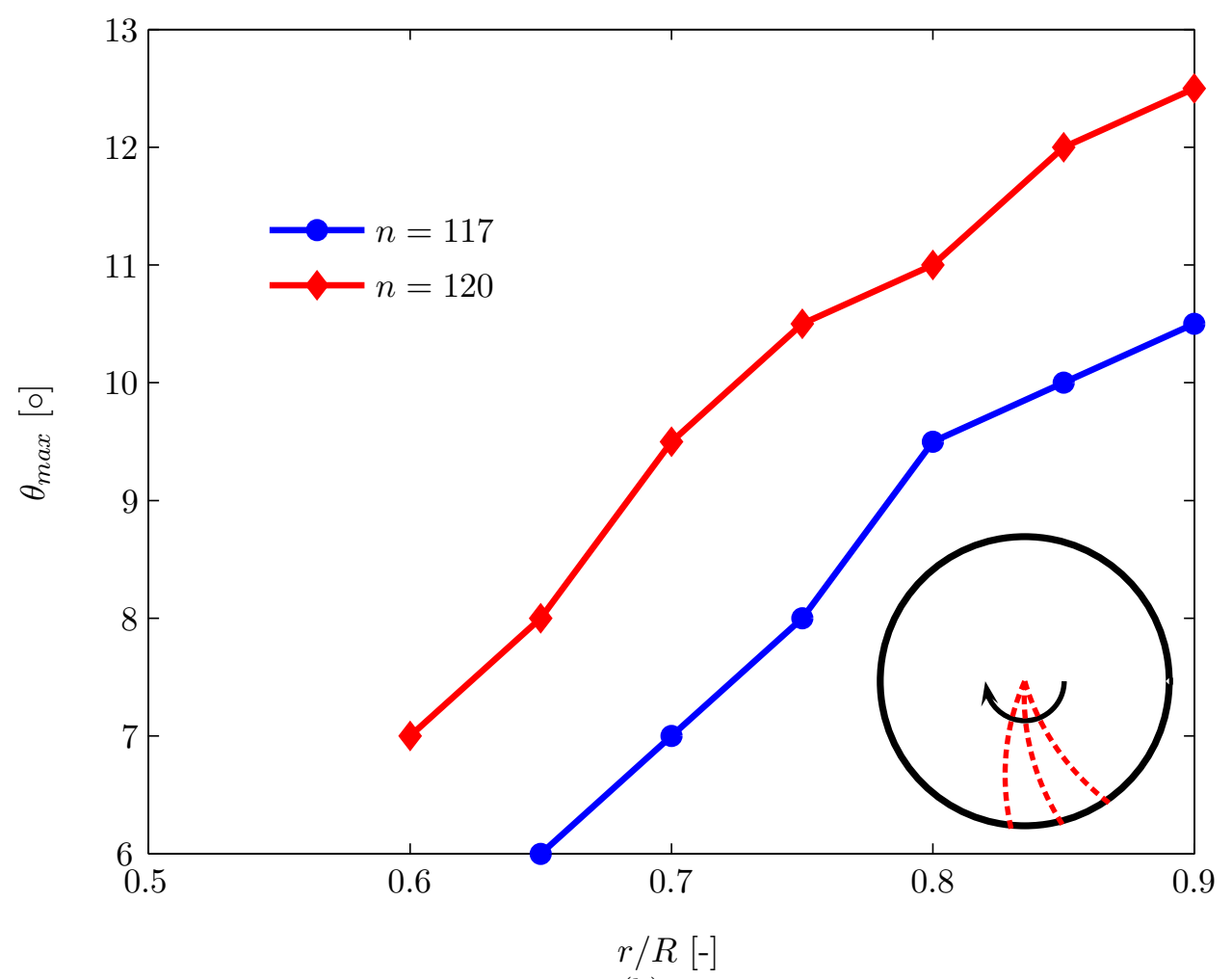

(b)

Figure 4: (a) Phase-averaged azimuthal profiles of the image brightness at $n=117$ for different radii $(r / R=$ $0.6-0.9)$; (b) Radial and azimuthal coordinates of the brightness peak for $n=117$ and $120\left(d_{o}=2 \mathrm{~cm}, h=3\right.$ $\mathrm{cm}, c=2.5 \mathrm{~g} / \mathrm{L})$. 

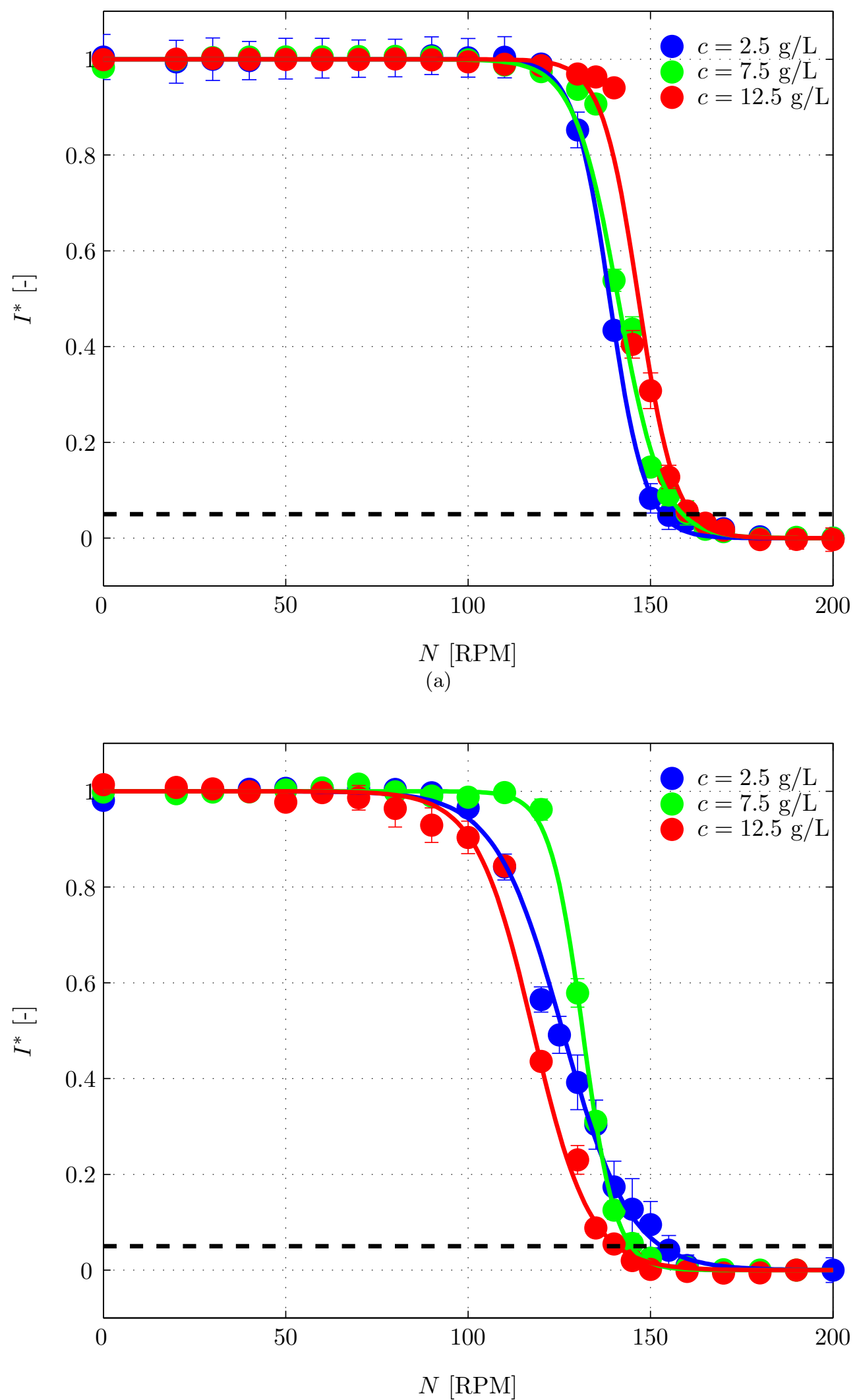

(b)

Figure 5: Variation of $I^{*}$ with shaker speed for different microcarriers' concentrations $\left(h=5 \mathrm{~cm}, d_{i}=7 \mathrm{~cm}\right)$ : (a) $d_{o}=1.5 \mathrm{~cm} ;(\mathrm{b}) d_{o}=2.5 \mathrm{~cm}$. 


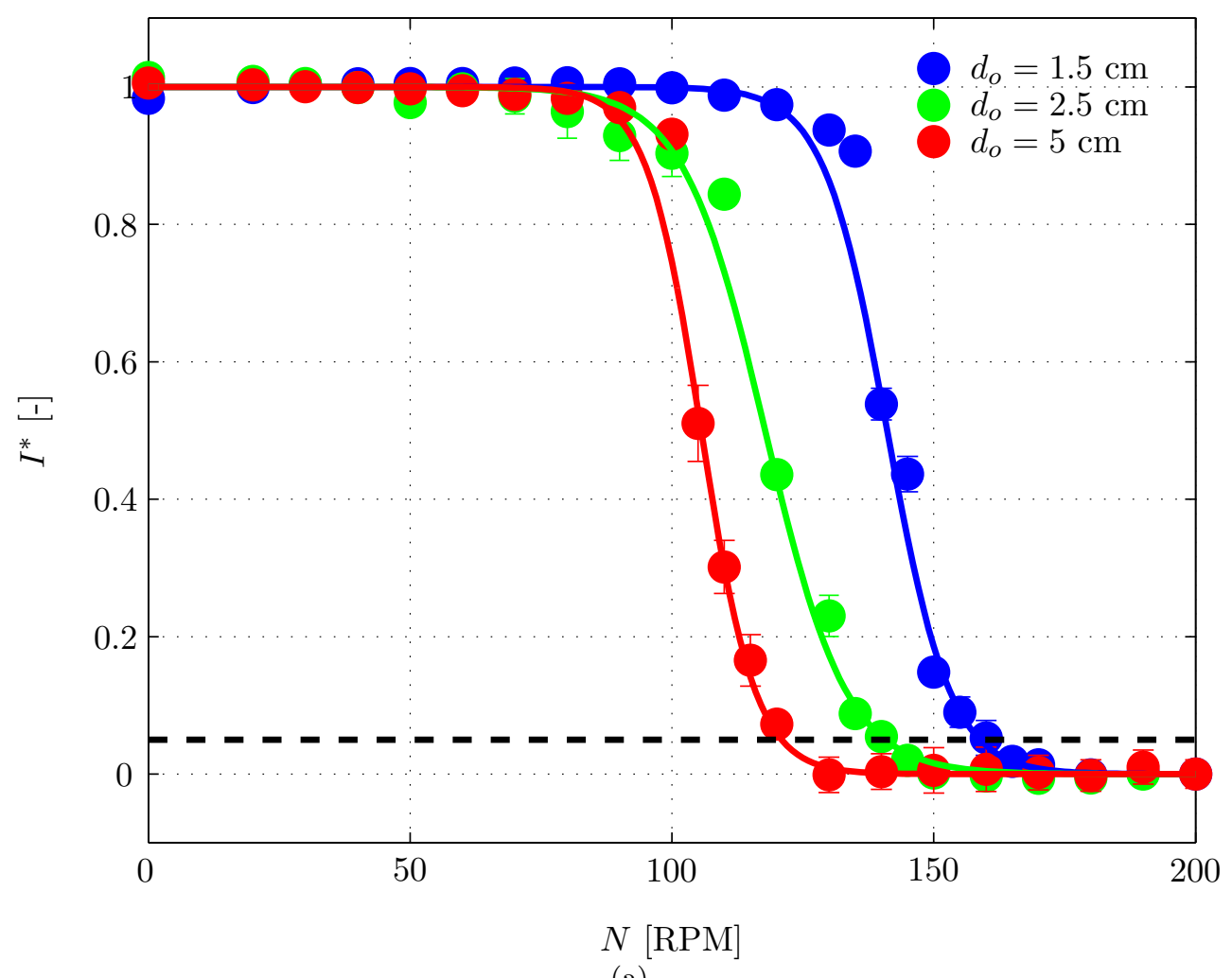

(a)

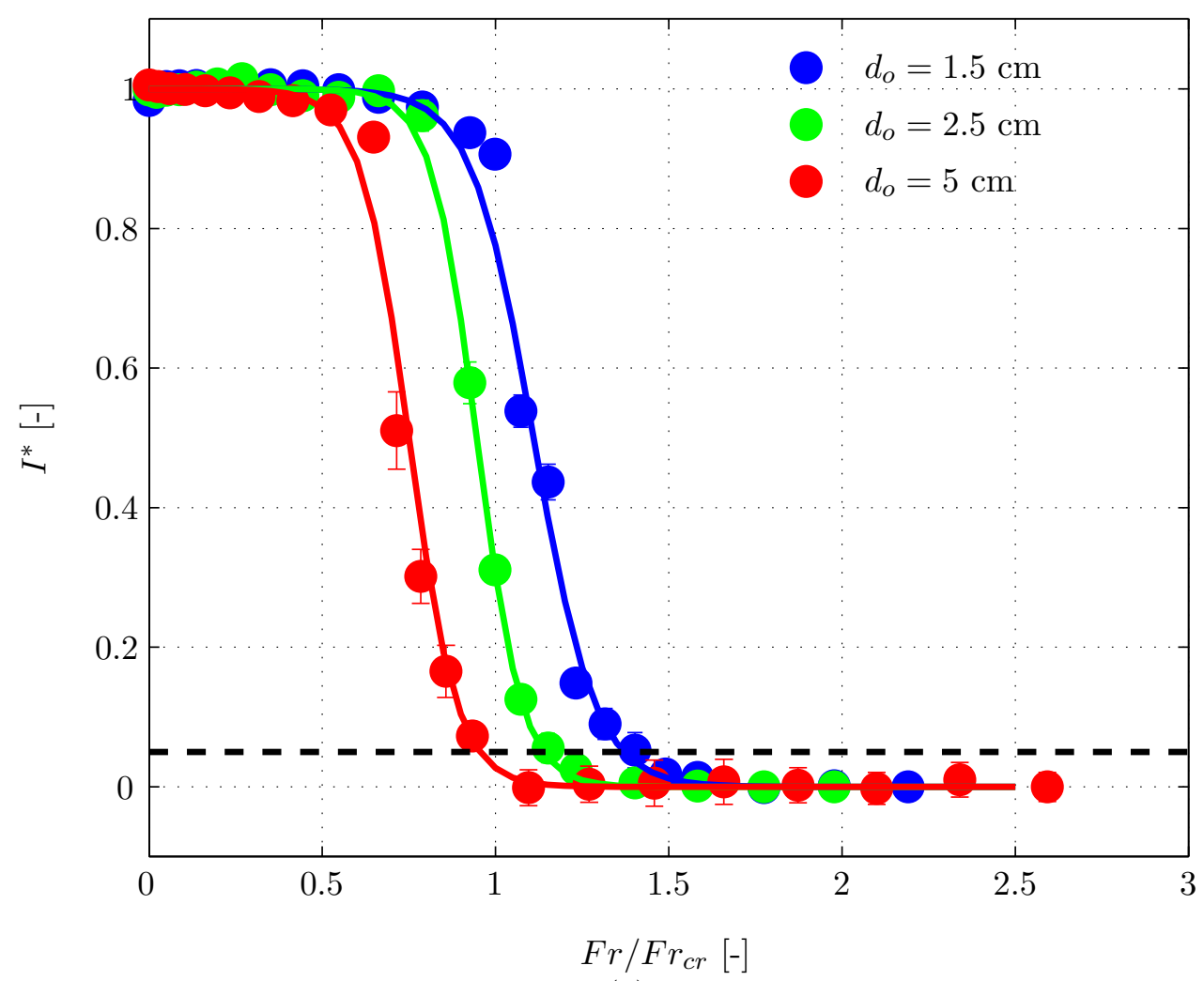

(b)

Figure 6: Variation of $I^{*}$ for different orbital diameters $\left(h=5 \mathrm{~cm}, d_{i}=7 \mathrm{~cm}, c=2.5 \mathrm{~g} / \mathrm{L}\right.$ ): (a) variation with shaker speed, $N$; (b) variation with $F r / F r_{c r}$. 

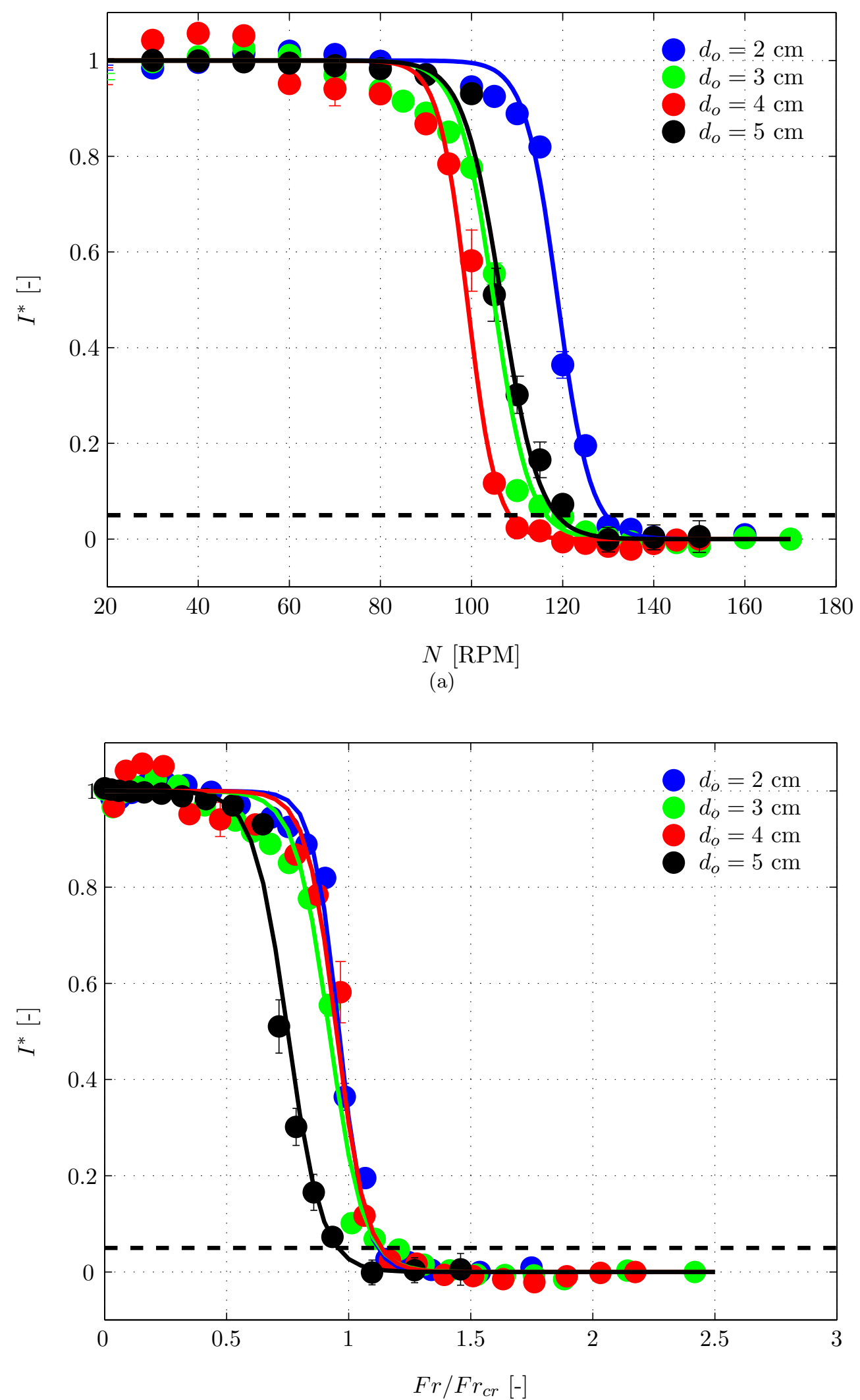

(b)

Figure 7: Variation of $I^{*}$ for different orbital diameters $\left(h=3\right.$ and $\left.5 \mathrm{~cm}, d_{i}=7 \mathrm{~cm}, c=2.5 \mathrm{~g} / \mathrm{L}\right)$ : (a) variation with shaker speed, $N$; (b) variation with $F r / F r_{c r}$. 


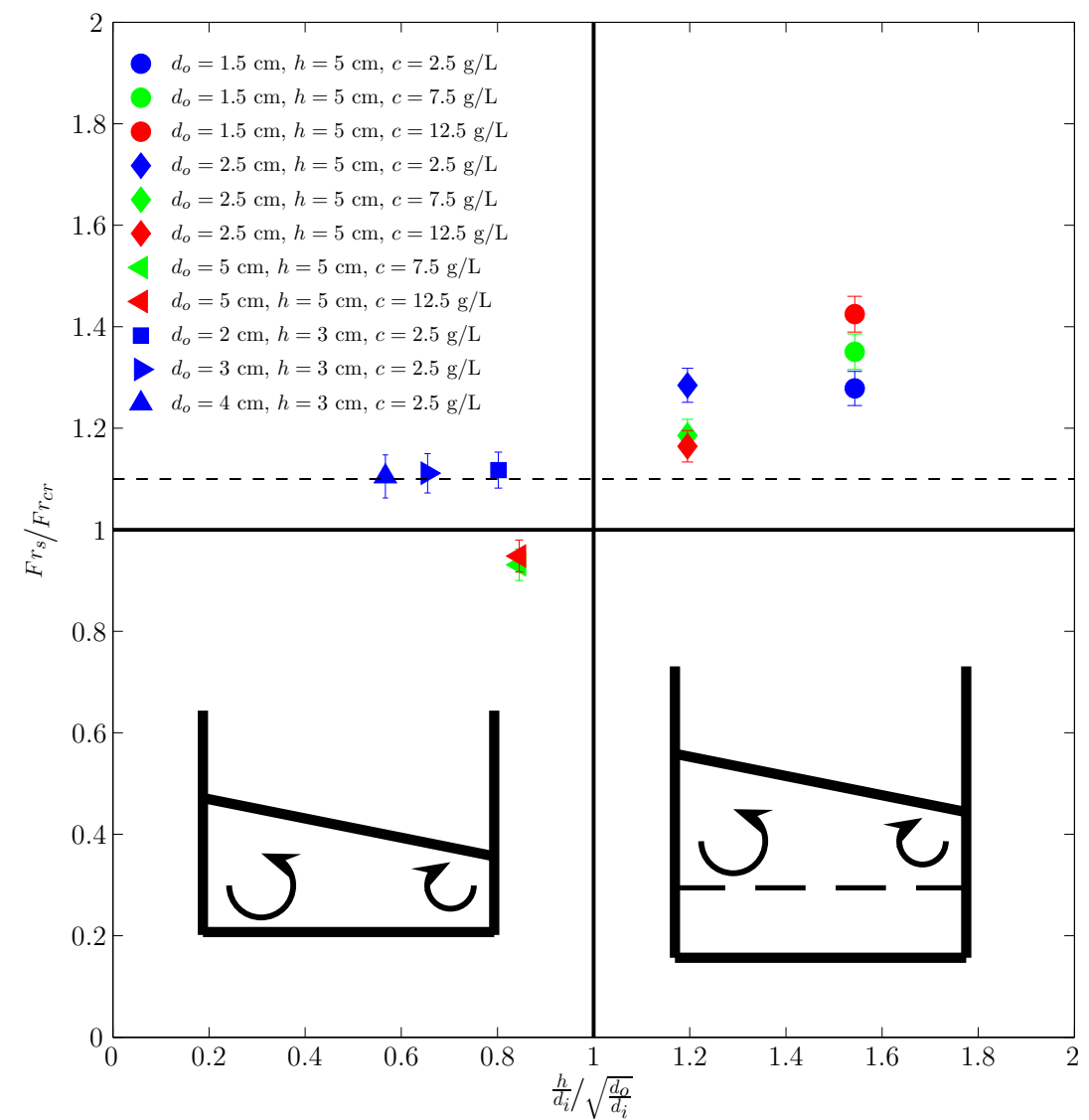

Figure 8: Variation of the suspended to critical Froude number ratio, $F r_{s} / F r_{c r}$, with critical height ratio, $\frac{h}{d_{i}} / \sqrt{\frac{d_{o}}{d_{i}}}$, for all the conditions investigated. 


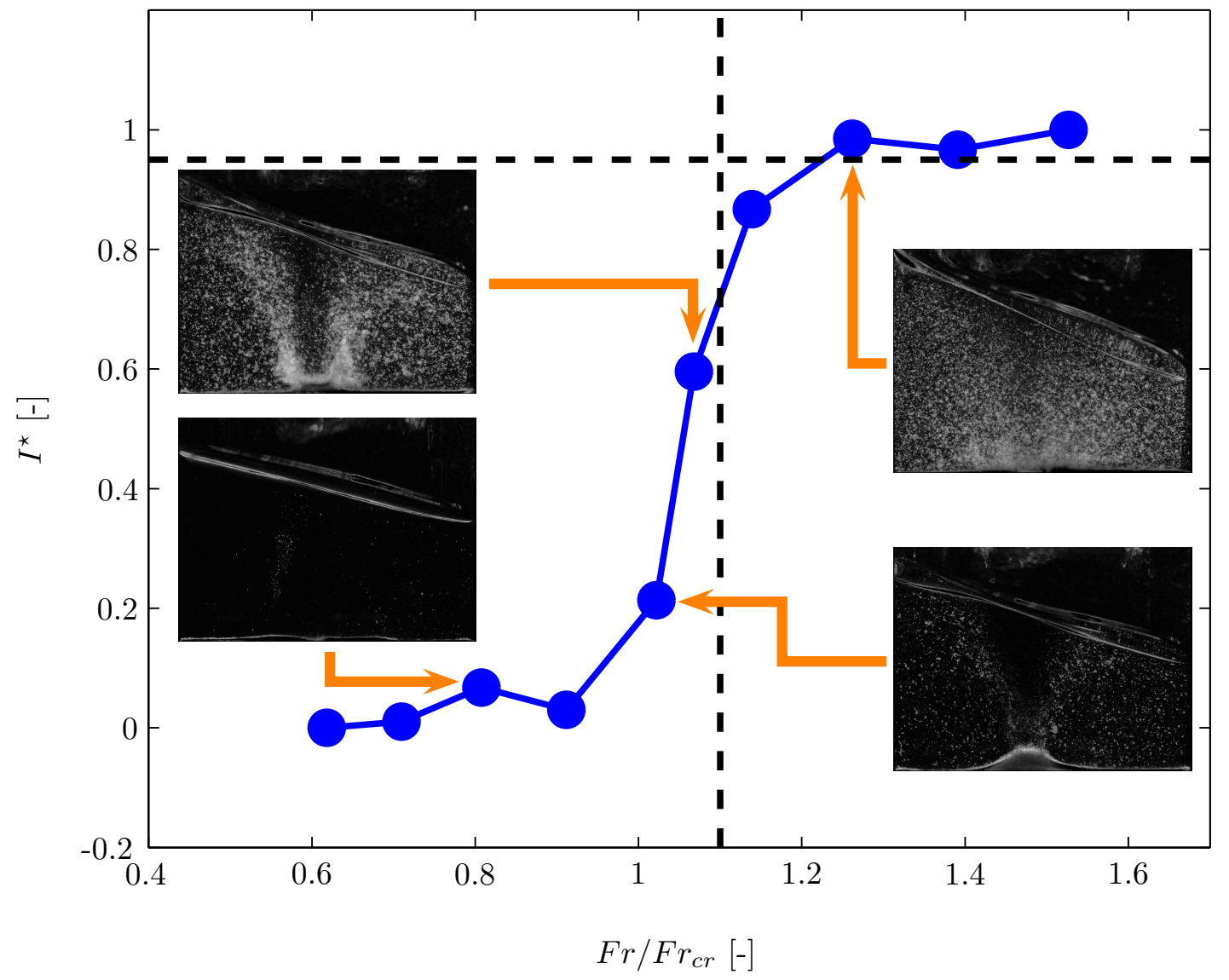

Figure 9: Variation of the normalised brightness index, $I^{*}$, with the Froude number ratio, $F r / F r_{c r}$, obtained from measurements on a vertical plane $\left(d_{i}=13 \mathrm{~cm}, d_{o}=5 \mathrm{~cm}, h=6.5 \mathrm{~cm}\right)$. 


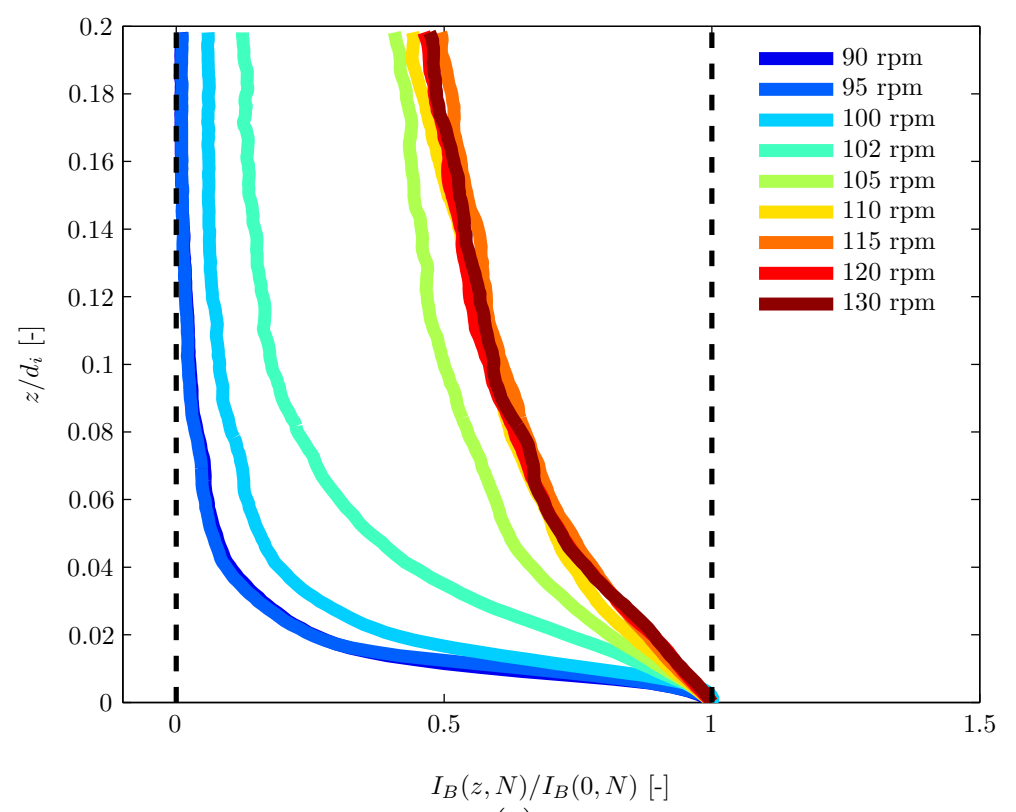

(a)

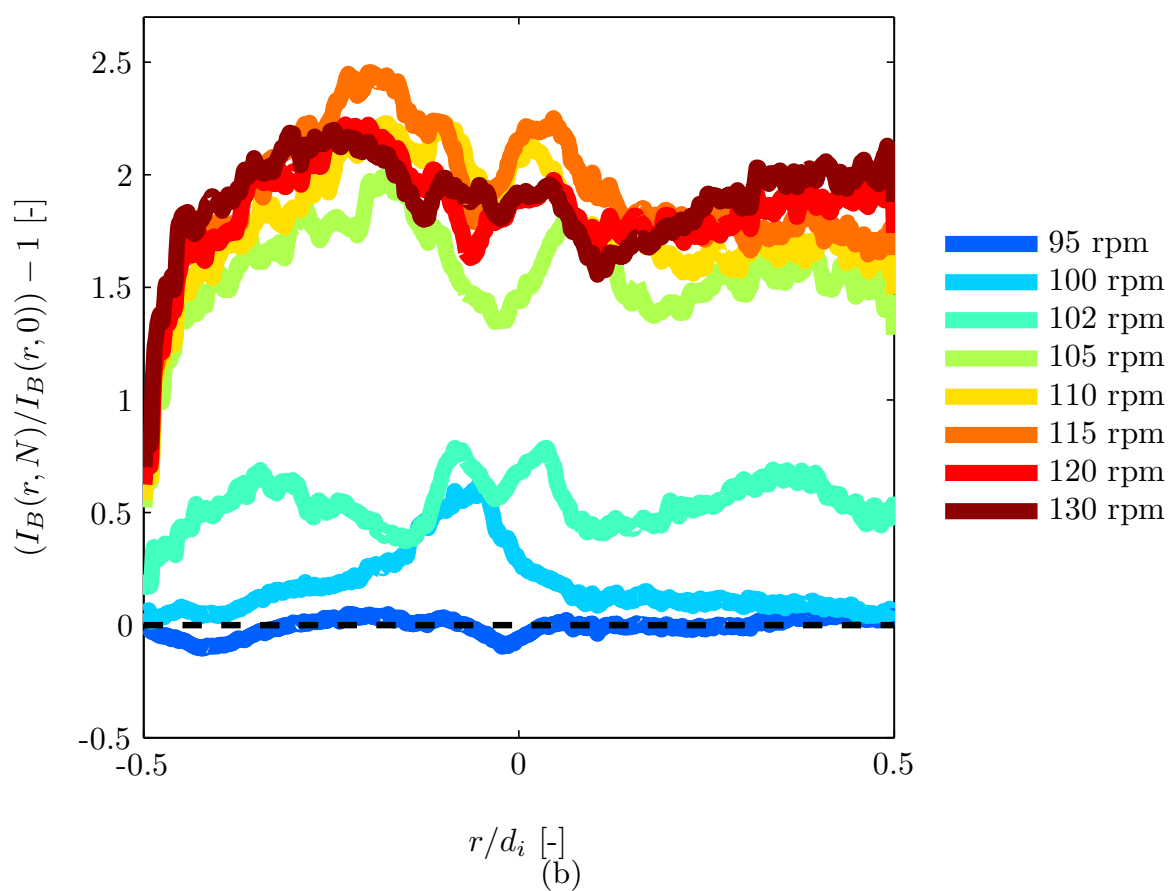

Figure 10: Profiles of the axial and radial cumulative brightness indices for increasing shaken speed $\left(d_{i}=10 \mathrm{~cm}\right.$, $d_{o}=5 \mathrm{~cm}, h=5 \mathrm{~cm}$ ): (a) Axial profiles; (b) Radial profiles. 


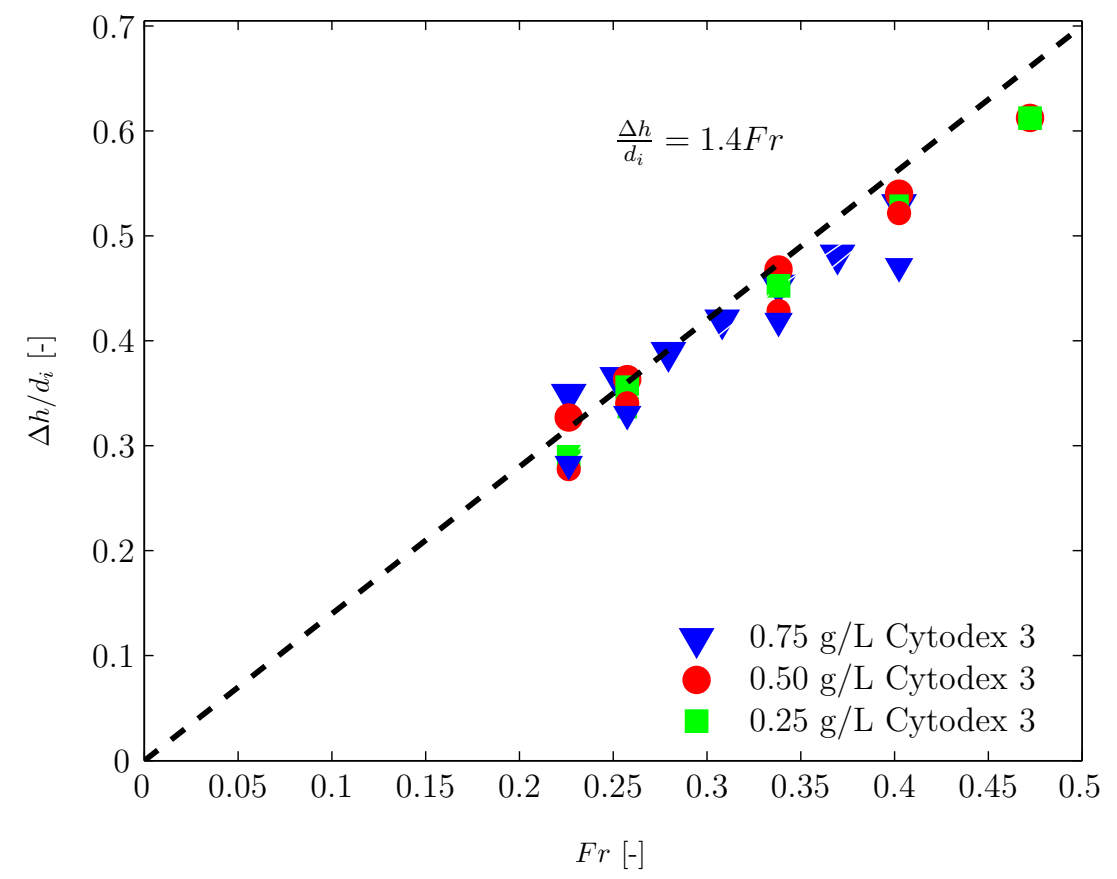

Figure 11: (a) Variation of the non-dimensional wave amplitude, $\Delta h / d_{i}$, with Froude number, $F r$, for different microcarriers' concentrations $\left(h=5 \mathrm{~cm}, d_{i}=10 \mathrm{~cm}, d_{o}=5 \mathrm{~cm}\right)$.

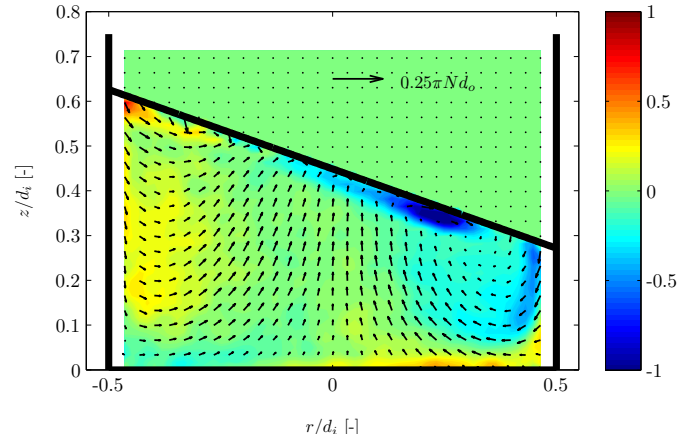

(a)

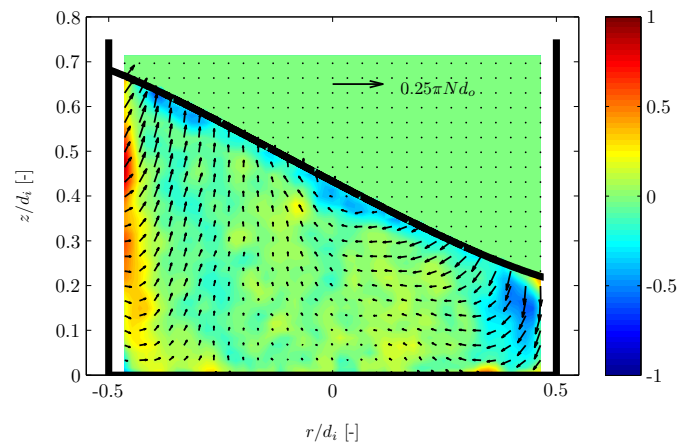

(c)

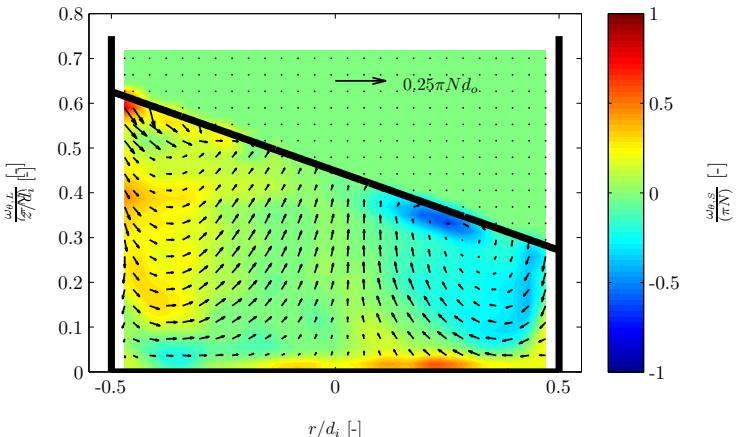

(b)

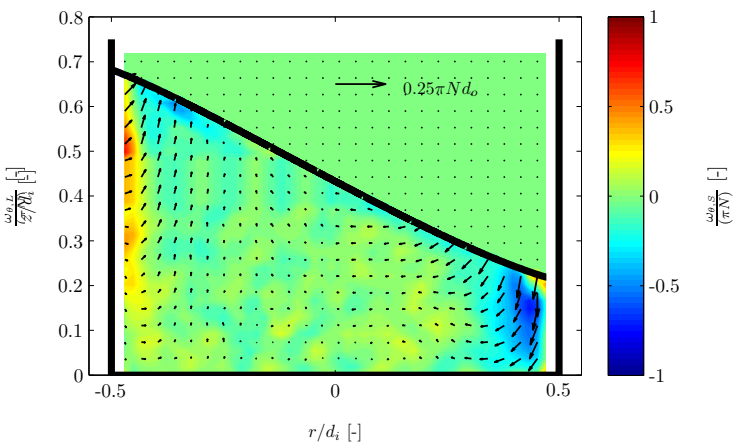

(d)

Figure 12: (a) Velocity vector fields and tangential vorticity contour maps of the liquid and solid phases before and after flow transition $\left(h=5 \mathrm{~cm}, d_{i}=10 \mathrm{~cm}, d_{o}=5 \mathrm{~cm}, c=0.5 \mathrm{~g} / \mathrm{L}\right.$ ): (a) Liquid phase, $N=90$ RPM; (b) Solid phase, $N=90$ RPM; (c) Liquid phase, $N=110$ RPM; (d) Solid phase, $N=110$ RPM . 